\title{
Hamiltonian Structure and Integrability
}

\author{
Benno Fuchsstciner \\ Cniversity of Paderborn \\ D 1790 Paderbom \\ Gemany
}

\section{Introduction}

Whenever a cuantity or a set of cluatities, evolves with time then we call this a dynamical system. The evolution of the universe certanty is a dynamical system, however a complicated one. The laws of evolution which gowern such a system are called the dynamical laws.

To describe dyanical systems we unally make suitable approximations in the hope of finding valid descriptions of their characteristic quantities. But even after such approximations we mostly canno write down explicitly how these quantities depend on time. usually such a dependence is much so complicated to be computed explicitly. Therefore we commonly write down dymanical systems in their infinitesinal form.

Considering a dyamical system in its infuitesinal form has many advantages. The principal one is that such an infinitesimal description is possible even in those cases where a global description is not feasible at all. Techuicaly speaking, an infinitesimal description leads to a differential equation. Which in many casces has monlinear terms due to the interaction between different quantities. To find such a differential exitation we only have to hnow a suitable set of dynamical laws. Howevet. solving such a noulinear differential equation for arbitrary starting points (intitial conditions) is often a liopelens endeavor.

Fortunatedy, the infinitesinal description sometimes gives an insight into the essential

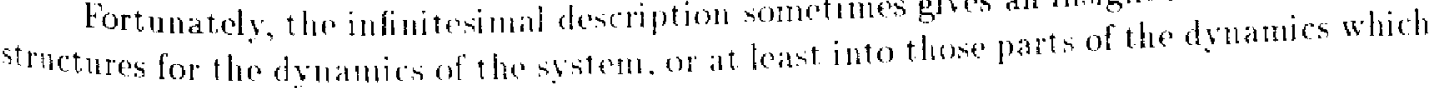
call be described localls.

Speaking from an abstact riewperint the maitl objects of our interest are explation of the form

$$
u_{t}=K(n)
$$

where $K(u)$ is a vector field on some manifold $h$ and where denotes the general point on this manifold. Since we to not rentrict the size of the dimension of the manifold $M$ this eguation still comprises an almudance of possible dynamical systems. For example $u$. could be the collection of all relcalul data of an economy, then equation (1.1) describes the evolution of that ceonomy. With regard to size of the manifold. this would be a rather simple dynanical systen since the manifold certainly has finite dimension whereas most

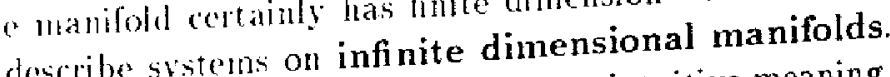
Most notions which later on with descly of cunation (1.1) do have a very intuitive meaning. For example. we call cquation (1.1) a flow on the underlying manifold. Thus we imagine that a point is flowing along its path on the manifold. Such a path is called an orbit of (u) describes the change in the position of $u$ for infinitesimal times, 
$K(u)$ must be tangential to the orbit of $u$. A simplified picture for the situation under consideration is:

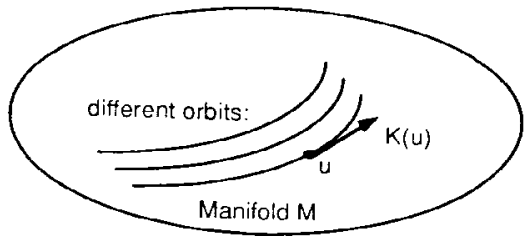

Fig. 1: Flow on a manifold $M$

Systems of particular importance are those describing the dynanics of particles in classical mechanics. For these systems the dyamical laws are determined by the total energy of the system. As an example, we consider the case when the energy $H=V+T$ is the sum of potential energy $V$ and linetic energy $T$, where $V=V\left(\vec{x}_{1}, \ldots, \vec{x}_{n}\right)$ only depends On the positions of the different particles and where the kinetic entergy is

$$
\tau=\frac{1}{2} \sum m_{i} \dot{x}_{i}^{2} . \quad\left(m_{2}=\text { mass of particle } i\right) \text {. }
$$

In order to elininate the masses $m_{i}$ (which are irrelevant for the structure of the system we introduce new coordinates in the space $\mathbb{R}^{2 n}$ (phant space)

$$
q_{i}=\vec{x}_{i} \cdot p_{i}=m_{i} \dot{\vec{x}}_{i}, i=1, \ldots, n .
$$

Lsing Newton's law we find

$$
\dot{q}_{t}=\frac{\partial H}{\partial p_{i}}, \dot{p}_{i}=-\frac{\partial H}{\partial q_{i}}
$$

or. if we introduce the formal field variable $\vec{u}$ to be the transposed of the position momentum vector $\vec{u}=\left(q_{1} \ldots \ldots q_{n}, p_{1} \ldots, p_{n}\right)^{T}$. then the dymamics has the form

$$
\vec{u}_{f}=\left(\begin{array}{cc}
0 & I \\
-I & 0
\end{array}\right) \Gamma H
$$

where $I$ is the identity in $11-\mathrm{space}$

Notable is that in this coluation only $\Gamma / /$ the grodiont of thre energy (taken in the Phase apace) enters. These erualions, dither in the form (1.3) or (1.4), are prototypes of

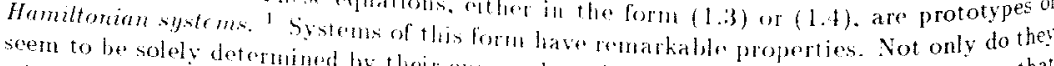

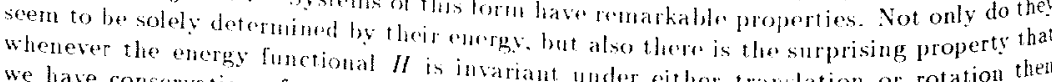
we lave conservation of monal $/ /$ is invariant under either iratistation or rotation then

or algular momentum, respectively. So there mus

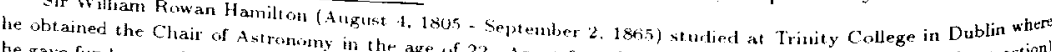

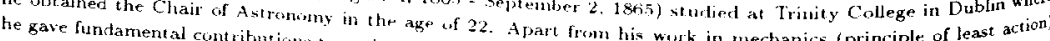
quaternions. 
be some kind of relation betwen the conservation laws of the system and its symmetry structure. Indeed such a mation war realed for classical Hamiltonian systems by Emmy Noether ${ }^{2}$ in her habilitation $[1,]^{3}$ and lhis relation is nowadays generalized to symmetries of so-called Lie-Bäcklund type for sysers on infinite dimensional manifolds. The special form of (1.3) or (1.1) is due to the special coordinate systen which was chosen, whereas the fundamental redation betwern symmetres and conserved quantities certainly nust go beyond a structure which is the romerpuence of a special coordinate system. So. in studying Hamiltonian equations the nunt be analwed in a differential geonetric invariant setup such that their structure becomesindependent of special rharts which were chosen to parametrize the underlying nanifold. We shall do tlat in the following sections 4 and 5 .

The best known cxamples of $l 1$ amiltonian systems probably ari2 the Harmonic Oscillator and the nonlincar pembilum, described below:

\section{Example 1.1: Harmonic Oscillator}

The evolution equations

$$
r_{i}=y \cdot y_{t}=-x \text {. }
$$

where $x(t), y(t) \in \mathbb{l} k$, describe 1 he time dependence of the hamonic oscillator. In matrix form this can be writom at

$$
\left(\begin{array}{l}
x \\
y
\end{array}\right)_{t}=\left(\begin{array}{cc}
0 & 1 \\
-1 & 0
\end{array}\right)\left(\begin{array}{l}
x \\
y
\end{array}\right) .
$$

which certainly has the form $(1.4)$ since

$$
\left(\begin{array}{l}
x \\
y
\end{array}\right)=\Gamma\|. \quad\|=\frac{1}{2}\left(x^{2}+y^{2}\right) .
$$

The manifold under consideration is $M=\mathbb{R}^{2}$. Int roducing the abbreviations

$$
h(u)=-u \cdot u=\left(\begin{array}{l}
x \\
y
\end{array}\right), . t=\left(\begin{array}{cc}
0 & 1 \\
-1 & 0
\end{array}\right)
$$

the evolution equation (1.5) in clealy an example for (1.1). Looking at this system we detect

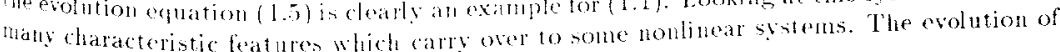
this flow is of the form

$$
(x p(t): u(0)-\cdots(t)
$$

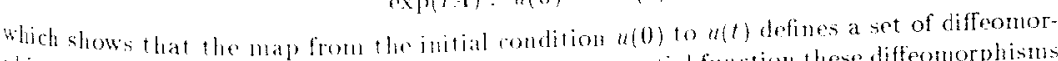

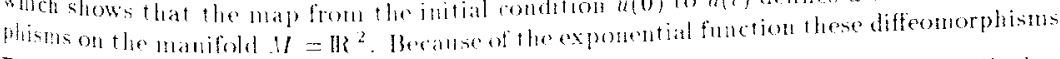

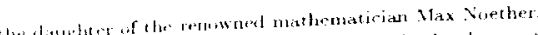

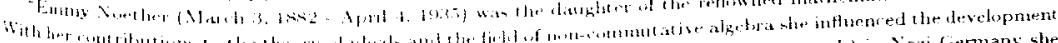

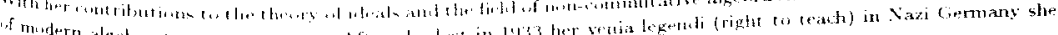

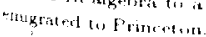

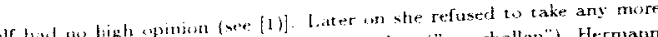

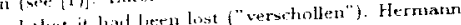

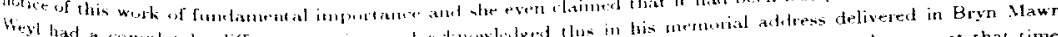

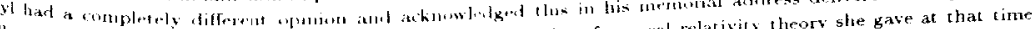

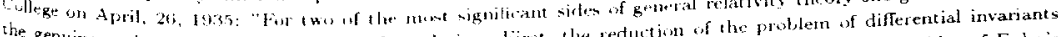

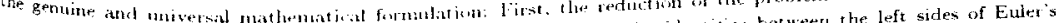

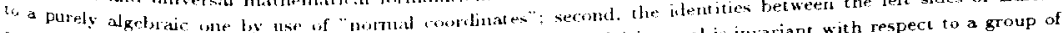

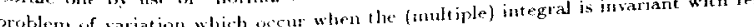
transformations.."
} 
form a representation of the additive group $(\pi,+)$. Furthemore, we observe the advantage of introducing polar coorditates $r=\sqrt{x^{2}+y^{2}}$ and $\varphi=\arctan (y / x)$. Then in this new coordinate space the system becomes a flow with constant velocity along the coordinate lines $r$ constant. Thus in this case we are able to split up the coordinates into two sets. one set (action variables) which remains constant under the flow, and another set (angle variables) which grows on the orbits linear with time. If such special coordinates having these properties can be introduced then we call such a system completely integrable. Look. ing back at our example we ses that this notion of complete integrability must be related to the existence of one-parameter symmetry groups. This is because changing one of the coordinates and leaving the others unchanged moves orbits into orbits. So this movement along coordinate lines constitutes a symmetry group.

\section{Example 1.2: Pendulum}

The time development in this rase is

Introducing

$$
\varphi_{t t}+\sin (\varphi)=0
$$

we see that (1.j) is of the form $q=\hat{r} p=\hat{r}$ and $\|=\left(\begin{array}{l}q \\ p\end{array}\right)$

we see that (1.3) is of the form $(1.1)$

$$
u_{t}=\left(\begin{array}{l}
q \\
p
\end{array}\right)_{t}=\left(\begin{array}{c}
p \\
-\sin (q)
\end{array}\right)=\left(\begin{array}{cc}
0 & 1 \\
-1 & 0
\end{array}\right)\left(\begin{array}{c}
\sin (q) \\
p
\end{array}\right) .
$$

The manifold nnder consideration again is $M=\mathbb{R}^{2}$. In contrast to (1.5) this equation constitutes a nonlinear fow. Again the dynamics has the form (1.4) since
. In contrast to (1.5)

$$
\left(\begin{array}{c}
\sin (q) \\
p
\end{array}\right)=\Gamma H . \quad H=1-\cos (q)+\frac{1}{2} p^{2} .
$$

Allough this in a montingar flow it can be linearized locally by introducing a suitable obvinate system. But this condinate system is no longer given by polar coordinates. given by the lines $I I=$ amstam ancl lines which we called action variables should now be How to do thin will be desmibed hit the remaining part should be chosen in a suitable way.

The soper of this artiche is

the hamonic oncillater antele is to replivase these simple observations which we made for more complicated sustem ingental framework so that they can be carried over to othet and relations in stacl a way that theyore, we wall to formulate the corresponding notions cloose

We organize the article in the following way: In the next section, we introduce some basic notions which lead in Section 3 to a description of the connection between symmetries and conserved quantities. It that point we shall not yet conoction between symmetries for the description. Instead of fommating everything in a differential geonetric invariant way we still will work with coordinate systems. This we do in order to keep the level of abstraction at the beginning as low as possible. Results in these sections are mosty 
presented without proofs because later on proofs will be given in a short and concise way by using a higher level of abstraction. In Section 4 we introduce Lie algebra modules, Lie derivatives and tensors in order to have a notation which allows one to see which notions are geometrically invariant. In Section 5 we introduce the notion of bi-hamiltonian fields on a general level. Then, in the following section, we introduce compatibility, especially for hamiltonian pairs, and illustrate the power of this notion by a set of suitable examples (Section 7). In the final part, Section 8 , we discuss complete integrability in the finite dimensional case and we show how that notion is connected to the situation considered before. In addition the action/angle structure of the multisoliton manifolds is given

\section{Basic Notions in Chart Representation}

I hope that most readers are accuainted with notions like manifolds, vector fields, tangent space, differentiability and so on. Ilowever. I do not believe that a knowledge of the theoretical background in manifold analysis is really necessary for understanding the concepts described in this article. For the most part a more intuitive grasp of infinitesimal calculus and a heuristic idea of manifolds as being something like smooth surfaces seems sufficient.

For the sake of completeness however, we include some remarks on this subject since notation will differ somewhat from the conventional notation, insofar as we avoid the calculus of exterior forms.

For infinite dimensional manifolds we will use the notion of Hadamard differentiability 26.] [27.] This is a fairly weak notion which nevertheless ensures the validity of the chain rlele. A function $F: E_{1}-E_{2}$ between two linear spaces ${ }^{4}$ is said to be Hadamarddifferentiable at $u \in E_{1}$ if there is a continuous linear map $L: E_{1}-E_{2}$ such that

$$
\lim _{\epsilon \rightarrow 0} \frac{1}{f}\{F(u+\epsilon v)-F(u)-c L[v]\}=0
$$

uniformly in $v$ on each compact subset of $E_{1}$. The linear operator $L$, and its application $L[y]$ to $v$ are then denoted by $F^{\prime}(u)$ and $F^{\prime}(u)[v]$, respectively. Of course, $F^{\prime}(u)\{v]$ is most easily computed from the directional derivative of $F$

$$
F^{\prime}[u]=F^{\prime}(u)[u]=\frac{\partial}{\partial \epsilon \mid c=0} F(u+\epsilon v)
$$

If not otherwise mentioned functions are usually assuned to be $C^{x}$-functions, i.e. infinitely often differentiable.

If the manifold is a vector space $M=E$, then vector fields are the continuous maps $K: E-E$ assigning to each $\| \in M$ some vector $K(u) \in E$. Again, we assume vector fields

\footnotetext{
- - Usually we do not

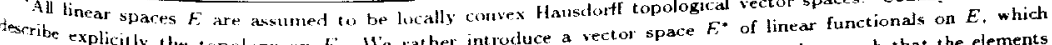
separate plicilly the topolugy on $E$. We rather introduce a vector space $F$ ef $E^{*}$ aroints, and we assiume that $E$ is endowed with the weakest locally con $E_{2}$ of linear maps $E_{1} \rightarrow E_{2}$ are then

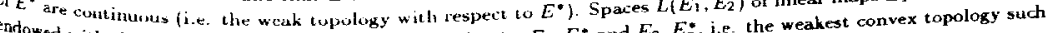
that with the weakeat lopology given by the dual pairs $E_{1}, E_{1}^{*}$ and $E_{2}, E_{2}^{*}$, i.e. the weakest convex continuous. all linear functionals $\mu$ on $L\left(E_{1}, E_{2}\right)$ given by $L \rightarrow \mu(L)=\rho(L(u))$ with $\rho \in E_{2}{ }^{\circ}, u \in E_{1}$ are conting
} 
to be $C^{\infty}$. Thus they constitute a Lie algebra with respect to the commutator defined by

$$
\begin{aligned}
{[K, G](u) } & =\frac{\partial}{\partial \epsilon \mid \epsilon=0}\left\{G\left(u+\epsilon K^{\prime}(u)\right)-K(u+\epsilon G(u))\right\} \\
& =G^{\prime}(u)[K(u)]-K^{-1}(u)[G(u)] .
\end{aligned}
$$

This Lie algebra is referred to as the vector field Lie algebra.

Recall that the definition of a Lie-algebra implies that the map $(K, G) \rightarrow[K, G]$ is bilinear, antisymmetric $([K, G]=-[G, K])$ and such that for all $K, G, L$ the Jacobi identity

$$
[[\kappa, G], L]+[[L, K], G]+\left[[G, L], K^{-}\right]=0
$$

holds. In fact this identity is easily verified by using the rhain rule of differentiation.

If the manifolds $M$ which we consider are not linear spaces, then derivatives are defined in the usual way by parametrizing. or modeling. manifolds by linear spaces. Although in most of our examples the underlying manifold is a vector spare we briefly illustrate that procedure for the sake of completeness. Those readers who do not care for technicalities should skip the following paragraphs up to the introduction of conserved quantities.

Let $M$ be some Hausdonff topological space and $E$ some linear space, then we call $M$ a $C^{x}$-manifold if there are given an open covering $\left\{U_{\alpha} \mid \alpha \in\right.$ some index set $\}$ of $M$ and homeonorphisms

$$
p_{\alpha}: V_{\alpha}-V_{\alpha}, V_{\alpha} \text { open susets of } E
$$

such that for all $\alpha$ and $f$ the overlap map $p_{\alpha} \circ p_{\beta}^{-1}$ is a $C \infty$ map $V_{\beta} \cap p_{\beta}\left(U_{\alpha} \cap U_{\beta}\right)-E$ These $p_{\alpha}$ can be considered to be local coordinates for the corresponding $l_{\alpha}$. The collection of these $\left(C_{\alpha}, p_{\alpha}\right)$ is defined to be an athas. Such an at las allows transfer of all aspects of the differential structure from $E$ to $M$. For example, a map $r$ on $M$ is defined to be $C^{\infty}$ if all the $=\circ p_{\alpha}^{-1}$ are $C^{x}$. Consider $u \in H$, then a chart around $u$ is a homeomorphism $p$ from an open neighhorhood $r$ of $u$ into the model space $E$ such that for all $\alpha$ the map $p o p_{x}^{-1}$ defined on $p_{0}\left(l^{\prime}\right) \cap b_{\alpha}$ is $C^{x}$. Now, the notion of tangent space is easily introduced. The The formal $T_{n} h$ at the proint $u$ is represented by the model space together with a charr. chosen tuence it mo has to be such that it does not depend on the special chart which is for fixed $u$. we const be given by equivalence classes with respect to different charts. 50. model space. In these pairs we $(\mu, v)$ consisting of a chart around $u$ and a vector $v$ in the by $\left.\left(p_{2} \circ p_{1}^{-1}\right)^{\prime}\left(p_{1}(a)\right)_{[} v_{1}\right]=1$. int roduce an equivalence relation $\left(p_{1}, v_{1}\right) \equiv\left(p_{2}, v_{2}\right)$ defined

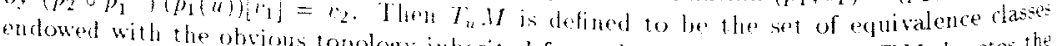
collection of all obcioss topologe inherited from the model space, and TM denotes the with these equibalese fallgent spacen and is called the tangent bundle. However, working commol represenace classes is not al ways very practical, so locally around $u$, we choose of the points aroutives by fixing some clart $p$ around $u$ and representing the tangent spaces $u \in M$ some element of to such a commont of $T_{u}, M$ is said to be a $C^{\infty}$-vector field if it is locally $C^{\infty}$ with respect

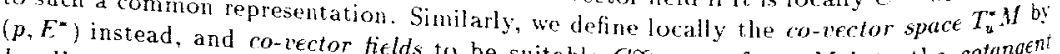
bundle (collection of all $T^{*}$. fields to be suitable $C^{\infty}$-maps from $M$ into the cotangent equivalence classes as all $T_{u}^{*} M_{\text {. }}$ ) Of course, strictly speaking, elements of $T_{u}^{*} M$ are again (only in the defuition above, one has to replace the derivatives 
by suitable adjoints in order to leave the application of a co-vector to a tangent vector invariant under coordinate changes). It should be observed that the choice of a common representation around some point $u \in U$ is the same as choosing a particular chart in the manifold given by the tangent bundle.

If the manifold under consideration is a linear space, then we do not really need all these constructions because wo thei model the manifold by jtself and for simplicity we choose the canonical chart given by the identity function on the model space. The validity of the requirements above then follows from the usual transformation formulas of differential calculus. In this case the tangent spaces $T_{u} M$ and cotangent spaces $T_{u}^{\prime *} M$ can be identified with $E$ and $E^{*}$, respectively, and we are back in the situation $M=E$ which we studied at the beginning.

We have chosen this formal approach to manifolds in order to indicate that differential calculus on abstract manifolds is indeed an easy task and that nevertheless for practical computations it mostly is sufficient to do analysis on linear spaces.

To proceed, we consider again

$$
u_{t}=K(u), u \in M, \quad M \text { some manifold. }
$$

$C^{x}$-maps from the manifold $M$ into the scalars (either $\mathrm{IR}$ or $(\mathbb{T}$ ) are called scalar fields. A scalar field $I(u)$ is said to be a conserved quantity for (1.1) if

$$
I^{\prime}(u)[H(u)]=0
$$

for all $u \in M$. The reason why this name has been chosen is obvious: Take an orbit $u(t)$ of (1.1), then by the chain rule we find

$$
\frac{d}{d t} I(u(t))=I^{\prime}(u(t))[K(u(t)]=0 .
$$

Hence, (2.5) guarantees that $I$ is constant along the orbits of (1.1).

Observe that. for every $u \in M$. the quantity $I^{\prime}(u)$ is a continuous linear functional on the tangent space $T, M$, ie $I^{\prime}(u)$ must be a cotangent vector. Derivatives of scalar fields are called gradients and $f$. Therefore we use for scalar quantities $I$ the notation $\nabla I(u)$ ilstead of $I^{\prime}(u)$. If we writo $>$ for the duality between tangent and cotangent vectors. then $(2.5)$ is written as

$$
\langle\Gamma I, K\rangle=0 \text {. }
$$

Sonetimes ther is some arlantage in looking at conserved quantities which depend explicilly on time. A family $f(u, t)$ of ecalar fields depending in a $C^{\infty}$-way on the parameter $t$ is satid to be a time dependent conserved quantity if

$$
F_{t}(u, t)+\langle\Gamma F(u, t), K(u)\rangle=0 .
$$

Here sub-t denoles partial derivative with respect to $t$ and $\nabla F=F^{\prime}$ is taken by ignoring the parameter $t$. The notion makes sense because it gives

$$
\frac{d}{d l} F(u(t), t)=0
$$


which implies that $F(u(t), t)$ is constant along the orbits of (1.1). From the physical point of view such a quantity does not seem to be very significant, since it is not invariant with respect to a translation of time. Nevertheless it turns out that it is a rather interesting quantity from the computational point of view.

Of special interest are those conserved quantities which are linear in $t$. Let

$$
F(u, l)=f_{0}(u)+f_{1}(u) t
$$

be such a quantity. Inserting $F$ into $(2.8)$ we then obtain by comparison of coefficients

$$
f_{1}+\left\langle\nabla f_{0}, K>=0 .\right.
$$

Hence, $F(u, t)$ is uniquely determined by its absolute term $f_{0}(u)$. Furthermore, the tern $f_{1}(u)$ must be a conserved quantity which is time independent.

Related to conserved quantities are one-parameter groups of $C^{\infty}$-diffeomorphisms on the manifold $M$. Recall that these are defined to be one-to-one $C^{\infty}$-maps such that the inverse is again differentiable. A one-parameter group of diffeomorphisms is a map $(u, \tau) \rightarrow R(\tau)(u)$ which is differentiable on the product $M \times \mathbb{R}=\{(u, \tau) \mid u \in M, \tau \in \mathbb{R}\}$ and assigns to every $\tau \in \mathbb{R}$ some diffeomorphism $R(\tau): M \rightarrow M$ such that

$$
R\left(\tau_{1}+\tau_{2}\right)=R\left(\tau_{1}\right)^{\circ} R\left(\tau_{2}\right) \text { and } R(0)=I
$$

for all $\tau_{1}, \tau_{2} \in \mathbb{R}$. This implies that all the $R(\tau)$ do commute and that $R(-\tau)$ must be the inverse of $R(\tau)$. With other words: $R(\tau)$ defines a group representation of the additive group $(\mathbb{R},+)$. One-parameter groups are completely determined by their $\tau$-derivative at $\tau=0$. To see this let $R(\tau)$ be a one-parameter group then

$$
G=\left.\frac{d}{d \tau} R(\tau)\right|_{r=0}
$$
is said to be its infinitesimal generator. Equation $(2.13)$ is an abbreviation for $G(u)=$
$(d / d \tau)\left\{\left.R(\tau)(u)\right|_{\mid \tau=0}\right.$. Since $R(\tau)$ assigns to each point of the manifold another point, $G$ must assign to each manifold point $z$ a tangent vector at $u$. Hence $G$ is a vector field. Because of the functional equation $(2.12)$ the $\tau$-derivative of $R(\tau)$ at arbitrary $\tau$ is easily
expressed by $G$ :

Hence $R(\tau)$ is uniquoty detorninted $\frac{d}{d T} R(\tau)=G^{\circ} R(\tau)$.

Hence $R(\tau)$ is uniquely determined by the vector field $C(u)$.

If the $R(\tau)$ are linear then $G$ again is linear. Then the solution of the linear differdiffeomorphism groups an formally be written as $R(T)=\exp (\tau(i)$. In general however. their structure is more or less given by equation (2.14) can be transformed inte exponential function since by use of pull-barks manifold with rather high dimension into a linear differential equation (on some abstract

To sce this.

of scalar fields. Let $R: M{ }^{\prime}=C^{\infty}(M, \mathbb{R})$ or $C^{x}(M, \mathbb{C})$, respectively, the vector space Let $R: M-M$ be a $C^{\prime \infty}$-map then

$$
\left(R^{-} f\right)(u):=f(R(u)), \quad f \in F
$$


defines a map $R^{*}: \mathcal{F} \rightarrow \mathcal{F}$ which is linear on $\mathcal{F} . R^{*}$ is said to be the pull-back given by $R$. Similarly, if $K$ is a vector field we define a map $L_{K}: \mathcal{F} \rightarrow \mathcal{F}$

$$
\left(L_{K} f\right)(u)=<\nabla f, K(u)>, \quad f \in \mathcal{F}
$$

by assigning to each $f \in \mathcal{F}$ its derivative in the direction $K . L_{K}$ is said to be the Liederivative given by $K$. Again, this is a linear map on $\mathcal{F}$.

The space of all Lie-derivatives is a vector space. The usual commutator of linear maps

$$
\left[L_{K}, L_{G}\right]=L_{K}{ }^{\circ} L_{G}-L_{G}{ }^{\circ} L_{K}
$$

endows this vector space in a natural way with a Jie-algebra structure.

Observation 2.1: The map $K \rightarrow L_{K}$ is a Lie-algebra isomorphism from the Lie algebra of vector fields onto the Lie-derivatives, i.e. we have

$$
L_{[K, G]}=\left[L_{K}, L_{G}\right]
$$

for all vector fields $K$ and $G$.

The proof is simple. since by differentiation we see that the commutator bracket is a representation of the vector field bracket. Moreover, the required fact that $L_{K} \neq L_{G}$ whenever $K \neq G$ follows from the observation that for any two different tangent vectors in $T_{u} M$ we can find a scalar field (by application of a suitable co-vector) having different derivatives in the direction of these tangent vectors. By similar arguments we find $R_{1}^{*} \neq R_{2}^{*}$ whenever $R_{1} \neq R_{2}$. Hence, it suffices to study the pull-backs and the Lie-derivatives instead of the original objects. For these new quantities equation (2.14) translates into

$$
\frac{d}{d \tau} R^{*}(\tau)=L_{K} R^{*}(\tau)
$$

which is clearly a linear differential equation since only linear operations are involved. There tore we write

$$
R^{*}(\tau)=\exp \left(\tau L_{K^{-}}\right)
$$

this obtaining a representation of the one-parameter group in terms of its infinitesimal generator. Of course, this is a highly artificial representation, since $R^{*}(\tau)$ and $L_{K}$ act on affinite-dinensional vector spaces even when $M$ is finite dimensional.

A consequence of these considerations is that one-parameter groups and evolition quations, whether linear or nonlinear, are more or less the same objects. To see this, let

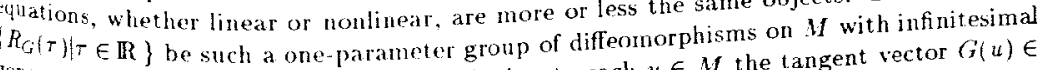
gerierator $(r$. Since $(r$ is a vector field assigning to each $u \in M$ the tangent vector $G(u) \in$ $T_{1 .}, H$ we look at evolution equation

In fact, for any initial condition $u(0)$ a solution is casily found, namely

$$
u(t)=R_{G}(t)(u(0)) .
$$


This solution for the initial value problem of $(2.21)$ must be unique since $R_{G}$ is a group: To see this take another solution $\bar{u}(t)$ fulfilling the same initial condition $\bar{u}(t=0)=u(0)$. Then by differentiation we obtain that $R_{G}(-t)(\bar{u}(t))$ inust be independent of $t$ and hence equal to $u(0)$. Recalling that $R_{G}(-\cdots t)$ is the inverse of $R_{G}(t)$ we find $\bar{u}(t)=u(t)$.

This viewpoint shows that $R_{G}(t)$ can be understood as the flow operator of (2.21) assigning to each initial condition $u(0)$ the solution $u(t)$ at time $t$. Of course, not all evolution cquations of the form (2.21) necessarily yicld one-parameter groups, only those where every initial condition $u(t=0)=u(0)$ have a unique solution for all $t$ such that the flow operator is a $C^{\infty}$-map.

This interpretation shows, that notions and methods coming from one-parameter groups must lead right-away to the crux of the algebraic aspects of evolution equations. Therein lies the problem of commutativity for nonlinear flows. It is easily seen that this iniportant property can be expressed in terms of infinitesimal generators. Looking at the exponcutial form of the pull-backs for these groups one discovers the infinitesimal equivalent for commutativity:

Observation 2.2: Let $R_{L_{L}}(\tau)$ and $R_{G .}(t)$ be two one-parameter groups of diffeomorphisms with infinitesimal generators $K$ and $G$. These two groups commute, i.e.

$$
R_{K}(\tau)^{\circ} R_{G}(t)=R_{G}(t)^{\circ} R_{K}(\tau) \text { for all } t \text { ard } \tau \text { in } I R
$$
if and only if $[\Lambda, G]=0$, i.e. their infinitesimal generators commute in the vector field
Lic-algebra.

In general, it is very hard to verify whether or not a vector field is really the infinites. nal generator of a one-parameter group because usually it is difficult to see if equation (2.21) has a unique solution for every initial coudition. But one of the reasons for the success of mathematical analysis is that global conditions (like existence and commutativity of groups $R_{k}(t)$ and $\left.R_{G}(\tau)\right)$ can be rephrased, by use of infinitesimal arguments, as local conditions. Therefore it scems natural to put the concept of symmetries onto a purely algebraic and infinitomimal basis by taking the commutativity of vector fields as definition (even in those (asws where $(2.21)$ is not the infinitesimal form of some globally defined group).

So. we define the vector field $G(u)$ to be a symmetry for the evolution equation (1.1) if and only if $[K,(]=$,0 . Here the notion syminetry is used as abbreviation for what corectly should be teruled as infinitesimal symmetry-generator.

Sote that when $(;$ is a symmetry for (1.1) then $K$ also is a symmetry for $u=G \mid u$. Csing the Jacobi identity we sere that whenever the vector fields $f^{\prime}$ and $L$ are symmetre for (1.1) then $[G, L]$ is again a skmmedry for this evolution equation. So, the symmetries of (1.1) are a subalgebra of the Lie al getria of vector fields.

It will turn out, that introduction of the concept of time dependent symmetries con parameter $t$ is said to be a time-dependent symmetry of $(1.1)$ if

$$
G_{t}+[K, G]=0 .
$$

Here, again $[K, G]$ is taken by ignoring the parameter $t$. 
If $G(u, t)$ and $L(u, t)$ are time-dependent symmetries then $[G, L]$ is again a timedependent symmetry. This is easily seen from (2.23) and the Jacobi identity. Hence, the time-dependent symmetries are again a subalgebra of the Lie algebra of vector fields.

The algebraic structure of time-dependent symmetries is very similar to the corresponding structure for conservation laws. For example if

$$
G(u, t)=G_{0}(u)+G_{1}(u) t
$$

is a time-dependent symmetry linear in $t$, then insertion into (2.23) and comparison of coefficients yields $G_{1}+\left[K, G_{0}\right]=0$. Hence $G(u, t)$ is uniquely determined by its absolute term $G_{0}(u)$. Furthermore, $G_{1}(u)$ must be a symmetry.

\section{Poisson Brackets and IIamiltonian systems}

If one compares equations $(2.8)$ and $(2.23)$ for the dynamical variables given by conserved quantities and symmetries one discowrs that these equations look very similar. They both are linear evolution equations on some infinite dimensional manifold

But there is one essential difference between these two equations. A difference which is easily discovered if one looks for means of constructing new solutions. 'A priori', equation (2.23) has more structure than equation (2.8) since there is a Lie algebra involved. This is of considerable advantage because we can take the commutator of any two solutions to find a new solution. So in order to complete the analogies hetween conserved quantities to look for lie algebra structure among solutions of es it seens intriguing to looking at the time derivative in both cases. The time ther viewpoint arises by looking a the the derivation, where derivation means is a special casc of what us all the Jacobi identity is a representation). Equation

2.23) tells us that this special time-derivative can be replaced by some inner derivation. derivation is sonething given by commutation with an element taken out der consideration And inner derivations are, from the mathematical if the structure under consideration. And inner dexample, apart from the discovery that vewpoint, much nicer than outer deriations. For example, apart from the reasons for the success of Hramical variables are operators rather than scalars, one of the reasonerators is given by glantum mechanics was the ansat? that the time evolution of theseuld have been feasible inier decivations. It is hard to imagine that quantum mechanics would have bet (1) in case of $(2.8)$ the tine derivative can be replaced by some inner derivation.

Fortunately, all these questions lead to the same structure, namely Hamiltonian systems. If one analyzes the situation further it all boils down to:

Problem 3.1: Take some operator calued function $\Theta(u)$ mapping ench manifold element $u$ Problem 3.1: Take some operator calued function $\Theta(u)$ mapping ench manifold ele $F_{1} . F_{2}$
to some linear operator $\Theta(u): T_{u} M^{*} \rightarrow T_{u} M$. Definc a bracket among scalar fields
$b_{y}$

$$
\left\{F_{1}, F_{2}\right\}_{\Theta}=\left\langle\nabla F_{2}, \Theta^{\circ} \nabla F_{1}\right\rangle \text {. }
$$


When is this a Lie-rlgebra? In addition, when is $\Theta^{\circ} \nabla$ a Lit algebra homomorphism into the vector field Lie algebra, i.e. when do we have

$$
\Theta^{\circ} \nabla\left\{F_{1}, F_{2}\right\}_{\Theta}=\left[\Theta^{\circ} \nabla F_{1}, \Theta^{\circ} \nabla F_{2}\right] \text { ? }
$$

We easily find the complete answer to that problem:

Theorem 3.2: The following are cquivalent:

(1) The bracket $\{,\}_{\Theta}$ defines a Lie algebra

(2) The bracket $\{\text {, }\}_{\Theta}$ defines a Lie algebra such that $\Theta^{\circ} \nabla$ fulfills (3.2), i.e. $\Theta^{\circ} \nabla$ is a Lie algebra homomorphism into the vector fields

(3) $\Theta$ has the following properties

(i) $\Theta$ is shew-symmetric with respect to the dhality between cotangent space and

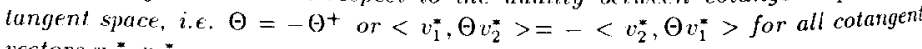
vectors $v_{1}^{*}, v_{2}^{*}$.

(ii) for all cotangent vectors $v_{1}^{*}, v_{2}^{*}, v_{3}^{*} \in T_{u} M^{*}$ the following identity holds $\left.<v_{1}^{*}, \Theta(u)^{\prime}\left[\Theta(u) v_{2}^{*}\right] v_{3}^{*}>+<v_{2}^{*}, \Theta(u)^{\prime}\left[\Theta(u) v_{3}^{*}\right] v_{1}^{*}>+<v_{3}^{*}, \Theta(u)^{\prime}\left[\Theta(u) v_{1}^{*}\right] v_{2}^{*}\right\rangle=$
0.

Proof:

First we show the equivalence between (1) and (3).

The skew-symmetry is certainly necessary and sufficient in order to guarantee that $\{.\}_{\Theta}$ is antisymmetric. Computation of the double-bracket vields

$$
\begin{aligned}
\left\{F_{1},\left\{F_{2}, F_{3}\right\}_{\Theta}\right\}_{\Theta}= & \left\langle\nabla\left\{F_{2}, F_{3}\right\}_{\Theta} \Theta \nabla F_{1}\right\rangle \\
= & \left.\left\langle\nabla<\nabla F_{3}, \Theta \nabla F_{2}\right\rangle, \Theta \nabla F_{1}\right\rangle \\
= & F_{3}^{\prime \prime}\left[\left(\Theta \nabla F_{2}, \Theta \nabla F_{1}\right)\right]-F_{2}^{\prime \prime}\left[\left(\Theta \nabla F_{3}, \Theta \nabla F_{1}\right)\right]+\left\langle\nabla F_{3}, \Theta^{\prime}\left[\Theta \nabla F_{1}\left[\nabla F_{2}\right\rangle\right.\right.
\end{aligned}
$$

Since second derivarives are symetric with respect to their entries all second derivatives $F^{\prime \prime}$ cancel if $\left\{F_{1},\left\{F_{2}, F_{3}\right\}_{\Theta}\right\}_{\Theta}+i t s$ ryclic permutations are taken. Therefore condition (3ji is equivalent to the Jacobi identity for $\{.\} \leftrightarrow$ which finishes the proof of the equivalence
between (1) and (3).

Since (2) implies (1) it only remains to prove that (3.ii) implies equation (3.2). To see this take two scalar fields $F_{1}, F_{2}$ and some arbitrary co-vector $v^{*}$. Since the secont derivatives of $F_{1} F_{2}$ are symmetric they all cancel in the following computation:

$$
\begin{aligned}
\left\langle v^{*},-\right. & \left.\Theta \nabla\left\{F_{1}, F_{2}\right\}_{\Theta}+\left[\Theta \nabla F_{1}, \Theta \nabla F_{2}\right]\right\rangle \\
= & \left\langle\nabla\left\{F_{1}, F_{2}\right\}_{\Theta,} \Theta v^{*}\right\rangle+\left\langle v^{*},\left[\Theta \nabla F_{1}, \Theta \nabla F_{2}\right]\right\rangle \\
= & \left\langle\nabla F_{2}, \Theta^{\prime}\left[\Theta v^{*}\right] \nabla F_{1}\right\rangle+\left\langle v^{*}, \Theta^{\prime}\left[\Theta \nabla F_{1}\right] \nabla F_{2}-\Theta^{\prime}\left[\Theta \nabla F_{2}\right] \nabla F_{1}\right\rangle \\
= & \left\langle\nabla F_{2} \Theta^{\prime}\left[\Theta v^{*}\right] \nabla F_{1}\right\rangle+\left\langle v^{*}, \Theta^{\prime}\left[\Theta \nabla F_{1}\right] \nabla F_{2}\right\rangle+\left\langle\nabla F_{1}, \Theta^{\prime}\left[\Theta \nabla F_{2}\right] v^{*}\right\rangle .
\end{aligned}
$$


Now recall that in our vector space situation every fixed co-vector $v^{*}$ is a gradient, a fact which is extremely easy to see: take the gradient of $\left\langle v^{*}, u\right\rangle$ to obtain $v^{*}$. Then application of condition $(3$, ii $)$ yields that the right hand side of this last equation es equal to zero. So we see that $\left\langle v^{*},-\Theta \nabla\left\{F_{1}, F_{2}\right\} \Theta+\left\{\Theta \nabla F_{1}, \Theta \nabla F_{2}\right]\right\rangle=0$. Moreover, because $v^{*}$ was arbitrarily chosen we obtain that the vector on the right side in this bracket is equal to zero, i.e.

$$
-\Theta \nabla\left\{F_{1}, F_{2}\right\}_{\Theta}+\left[\Theta \nabla F_{1}, \Theta \nabla F_{2}\right]=0
$$

which shows that $\Theta \nabla$ is a Lie algebra homomorphism.

Operators $\Theta$ having one of the equivalent properties of the last theorem are called implectic operators or Poissson operators, they play a fundamental role for dynamical svstems. The corresponding bracket introduced in (3.1) then is termed Poisson bracket. The flow

$$
u_{t}=K(u)
$$

is called a hamiltonian flow ${ }^{5}$ if there is some scalar field $H(u)$ and some implectic operator $\theta(u)$ such that

$$
u_{t}=\Theta(u)^{\circ} \nabla H(u) \text {. }
$$

The scalar field $H$ then is the so called Hamiltonian of the system. The Poisson brackets give a suitable frame for describing the dy namics of scalar fields with respect to the evolution given by (1.1). Using $K=\Theta \nabla H$ we find that the total time derivative of some $F=$ $F(u(t), t)$ can be written as

$$
\frac{d}{d t} F=\{\nabla H, F\}_{\Theta}
$$

Hence a scalar field is a conserved quantity if and only if it commutes (in the Lie algebra given by the Poisson brackets) with the hamiltonian of the flow. As a particular consequence of that we have that the lamiltonian $H$ itself always is a conserved quantity. This quantity usually is called energy ${ }^{6}$. However, the most important consequence of the above theorem is that now we have a precise relation between conserved quantities and symmetries:

Theorem 3.3: Whenever $I(u)$ is a conserved quantity for the hamiltonian flow $u_{t}=$ $\theta i)^{\circ} \nabla H(u)$ then $\Theta^{\circ} \nabla 1$ is a symmetry of that flow.

Proof: By Theorem 3.2 we have $[K, \Theta \nabla I]=\Theta \nabla\{H, I\}_{\theta}$. This expression is equal to zero since $l$ is a conserved quantity:

This result we call Noether s theorem since it is a generalization of the classical result abtained by Emmy Noether ([18)]. A simple exercise shows that it carries over to timedependent conserved quantities and time-dependent symmetries as well.

Example 3.4: Pendulum

If the manifold is a vector space and $\Theta$ an antisvmmetric operator which does not depend on the manifold point $u$ then we obviously have $\Theta(u)^{\prime}=0$, hence $\Theta$ fulfills condition 3 of

\footnotetext{
istuation for hamitonian flows it is uslly required that $\theta$ is invertible. But

anifolds where, for topological reasons, invertibility is a little

bit problematicing interested in infunte dimensional manico condition.

more than one hamiltonian formit careful wit
} 
Theorem 3.2 and therefore must be implectic. A particular example for such an operator is the antisymmetric matrix appearing in equations (1.5) and (1.7). So, these equations give hamiltonian formulations for these systems and, as stated above, their hamiltonians are given by energy conservation.

It should be mentioned that whenever the manifold is finite dimensional and the implectic operator $\Theta$ is invertible then locally there is a coordinate transformation on the manifold such that in the new coordinates the implectic opcrator is an off-diagonal matrix having -1 's in the upper half and +1 's in the lower half of the off-diagonal (see ([16, page $30])$ ). This means that equations (1.3) represent the prototype of hamiltonian equations in finite dimension.

The importance of conserved quantities is seen from the fact that even for a nonlinear system like the pendulum knowing the energy allows to integrate that equation completely To see this we first remark that we already know the orbits in phase space, since they are given as $H=$ constant

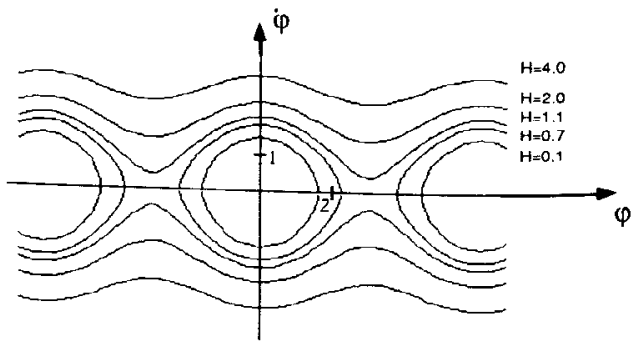

Fig. 2: Phase space orbits of the pendulum To integrate the equation along these orbits we choose a fixed value $E$ for this conserved
quantity, then

is a differential equation of first order and separation of variables yields that

$$
\begin{aligned}
& H(\dot{\varphi}, \varphi)=\frac{1}{2} \dot{\varphi}^{2}-\cos (\varphi)=E \\
& \text { rst order and separation of variables yiel } \\
& \int \frac{d \alpha}{\sqrt{2 E+2 \cos (\alpha)}}=\text { constant }=F
\end{aligned}
$$

must be a constant. This formula obviously gives the solution of (1.7) in implicit form Expressing $E$ again by $H(\dot{r}, \vec{r})$ we obtain that

$$
F=t-\int \frac{d \alpha}{\sqrt{2 H(\dot{\varphi}, \varphi)+2 \cos (\alpha)}}
$$
must be constant along any line on which $H(\dot{\varphi}, \varphi)$ is constant. Hence $F$ is constant along
the orbits of $(1 . j)$. Rewriting, this in phase-space variables we have found a time-dependent
conserved conserved quantity for $(1.8)$. 
The pendulum provides the most simple which illustrates that knowing suitable and enough conserved quantities implies that an equation can be integrated.

Example 3.5: Korteweg-de Vries equation

The Korteweg-de Vries equation [13(]KdV for short)

$$
u_{t}=K(u):=6 u u_{x}+u_{x x x}
$$

plays a central role in the history of completely integrable systems on infinite dimensional manifolds.

Usually, this equation is considered to be a flow on the space $S$ of tempered functions. These are the $C^{\infty}$-functions $f$ in the real variable $x \in \mathbb{R}$ having the property that $f$ and all its derivatives vanish at $\pm \infty$ faster than any rational function.

Define $\mathcal{S}^{*}$ to be the space of $C^{\infty}$-functions in $x$ such that all derivatives grow at most polynomially at $\pm \infty$. This space can be taken as a space of linear functionals on $\mathcal{S}$ by using an $L^{2}$ scalar product in the following way . namely

$$
\langle U, u\rangle=\int U(x) u(x) d x, \quad U \in S^{*}, u \in S .
$$

We use the convention that if no boundaries are given then integrals always go over $\mathbb{R}$ u: $\mathbb{R}^{n}$, respectively. As topology we take the woakest comvex topology making all these functionals continuous. Then the scalar ficlds

$$
\begin{gathered}
I_{0}(u)=\int u(x) d x \\
I_{1}(u)=\int u(x)^{2} d x \\
I_{2}(u)=\int\left(u^{3}-\frac{1}{2} u_{x}^{2}\right) d x
\end{gathered}
$$

are $\left(x^{x}\right.$ functions $M \rightarrow \mathbb{R}$. The derivative of, for example, $I_{2}(u)$ is computed to be

$$
I_{3}^{\prime}[v]=\left.\frac{\partial}{\partial \epsilon}\right|^{c=0} \int\left\{(u+\epsilon v)^{3}-\frac{1}{2}(u+\epsilon v)_{x}^{2}\right\} d x
$$

Integration by parts yields

$$
I_{3}^{\prime}[r]=\int\left(3 u^{2}+u_{x x}\right) x d x
$$

Hence provided the duality between tangent and cotangent space is represented by (3.9). then the gradient of $I_{2}$ can be identified with $3 u^{2}+u_{x x}$. The gradients of $I_{0}, I_{1}$ are given in the same way by the functions 1 and $2 u$, respectively. We compute $I_{1}^{\prime}[K(u)]$ for $K(u)$, the

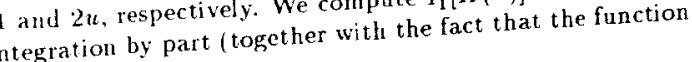
$u$ is tempered) yields

$$
I_{2}^{\prime}(u)[\boldsymbol{K}(u)]=\int 2 u\left(u_{x x x}+6 u u_{x}\right) d x=2 \int\left(2 u^{3}+u u_{x x}-\frac{1}{2} u_{x}^{2}\right)_{x} d x
$$


The latter expression is equal to zero because the integrand is the derivative of a tempered function. So, $I_{1}$ is constant along the orbits of the $\mathrm{KdV}$. The same holds true for the ficlds $I_{0}$ and $I_{2}$, whence all these quantities are conserved for the $\mathrm{KdV}$. A time-dependent conserved quantity is for example

$$
F(u, t)=\int\left\{x u-3 t u^{2}\right\} d x .
$$

We can write the Korteweg-de Vries equation in the following way

$$
u_{t}=\Theta^{\circ} \nabla H
$$

where

$$
\Theta=D \text { differential operator with respect to } x
$$

and

$$
H=\int\left(u^{3}-\frac{1}{2} u_{x}^{2}\right) d x .
$$

Since $\Theta$ is an antisymmetric operator which does not depend on the manifold points. $\theta$ must be implectic and this is a hamiltonian formulation. So one is inclined to call $H$ the energy of the systen. However, another way to write the KdV is the following

$$
u_{t}=\Theta^{\circ} \nabla H
$$

where

and where

$$
H=\frac{1}{2} \int u^{2} d x
$$

$$
\Theta=D^{3}+2(D u+u D)
$$

is again antisymnetric and is shown to fulfill condition (3. ii) of Theorem 3.2 (see Section i). Hence this is a second hamiltonian formulation for the $\mathrm{KdV}$

This last example shows that for some systems hamiltonian formulations are not unique. It will turn out, however, that this non-uniqueness is a highly desired propert? which will help to construct suitably many conserved quantities and thus will enable $u$
to integrate the equation. The main idea for generating infinitely many conservation laws
from two different hamilton from two different hamiltonian formulations goes back to F. Magri ([14)] who propostu that one hamiltonian formulation should be used for going from a conserved quantity to symnetry and then by the second one one should go back to another conserved quantity Thus an infinite sequence of conservation laws would be generated. There is one apparent difficulty with this concept, namely, that the map $\Theta \nabla$ is not invertible. This difficult: is overcome by transferring the result stated in Theorem 2.3 to co-vector fields instead of scalar fields. Then instead of going back to scalar fields one goes back with the hamiltonia? formulation to co-vector fields instead and from there, by integration, to the corresponding potentials. Of course, for doing that one requires that the co-vector fields generated this way are closed (a notion which we will introduce in the next section). This requirement in Section 6). A nother closed co-vector fields will lead to the notion of compatibility (treated boundary conditions at difficulty with this concept arises already for the $\mathrm{KdV}$ when othes 
anymore for this equation since the integrals $(3.10)$ to $(3.12)$ then clearly diverge. In order to subsume even this case under a common theory we have to lift all our notions to a new level of abstraction. This new level of abstraction, which will be formulated in the next section, then provides a more transparent setup so that the necessary considerations can be carried out more easily.

\section{Lie derivatives}

In this section we would like to review the basics of symplectic geometry and Hamiltonian merhanics on an abstract level. This high degree of abstraction will enable us to represent the relevant results in a very concise way.

Let $\mathcal{F}$ be some commutative algebra (over $\mathbb{R}$ or $\mathbb{C}$ ) with identity. We now assume $\mathcal{L} .[], \mathcal{F})$ to be a Lie-module $([17)]$. Recall that being a Lie module means that $(\mathcal{L},[])$ is a Lie algebra such that a multiplication between elements of $\mathcal{L}$ and $\mathcal{F}$ is defined and that, furthermore, there is a canonical homomorphism

$$
\kappa \rightarrow L_{K}
$$

from $\mathcal{L}$ into the derivations on $\mathcal{F}$. For $K, G \in \mathcal{L}$ and $f \in \mathcal{F}$ these derivations have to fulfill

$$
\begin{gathered}
{[K, f G]=f[K, G]+L_{K}(f) G} \\
J_{K} L_{G}-L_{G} L_{K}=L_{[K, G]} .
\end{gathered}
$$

Of course, being a derivation on $\mathcal{F}$ means that the product rule

$$
L_{K}(f g)=L_{K}(f) g+f L_{K}(g) \text { for all } f, g \in \mathcal{F}
$$

holds. In the following we require, for technical reasons, that the map $K \rightarrow L_{K}$ is injective.

Lie modules are the cononical extensions of Lie algebras admitting a lie nlgebra homomorphism into the derinations of $\mathcal{F}$. To be precise: Let $\mathcal{L}_{1}$ be some Lie algebra rontaned in some $\mathcal{F}$ and such that $\mathcal{L}$ is the linear hall of $\left\{f h^{\circ} \mid f \in \mathcal{F}, K \in \mathcal{L}_{1}\right\}$. Then into the derivations of $\mathcal{F}$ is given then there is a unique extension of $\left(\mathcal{L}_{1},[]\right)$ into a Lie module structure $(\mathcal{L},[], \mathcal{F})$ such that $(4.1)$ ard (4.2) hold.

The proof of this remart is a simple computation. One takes $K_{1}, K_{2} \in \mathcal{L}_{1}$, then makes the strions definition

$$
\left[f_{1} K_{1}, f_{2} K_{2}\right]:=f_{1} f_{2}\left[K_{1}, K_{2}\right]+f_{1} L_{K_{1}}\left(f_{2}\right) K_{2}-f_{2} L_{K_{2}}\left(f_{1}\right) K_{1} .
$$

aild the extension to all of $\dot{L}$ is obtained by taking sums.

and $\mathcal{F}$ are given then we assume that all quantities introduced below are continueus. $\mathcal{F}$-linear functionals $\gamma: \mathcal{L} \rightarrow \mathcal{F}$ we denote the application of $\because$ to $K \in \mathcal{L}$ by $\langle\gamma, K\rangle$. Such a functional $\gamma$ is said to be closed if

$$
L_{K}\left\langle\gamma, G>-L_{G}\langle\gamma, K>=\langle\gamma,[K, G]>\text { for all } G, K \in \mathcal{L} .\right.
$$


For $f \in \mathcal{F}$ we denote by $\nabla f$ the special $\mathcal{F}$-linear functional on $L$ given by

$$
\left\langle\nabla f, K>:=L_{K} f \text { for all } K \in \mathcal{L}\right. \text {. }
$$

Because of (4.2) all these $\nabla f$ are closed. A suitable $\mathcal{F}$-module of $\mathcal{F}$-linear maps $\mathcal{L} \rightarrow \mathcal{F}$ generated by closed $\mathcal{F}$-linear functionals $\gamma: \mathcal{L} \rightarrow \mathcal{F}$ is denoted by $\mathcal{L}^{*}$. We assume that $\mathcal{L}^{*}$ contains all $\nabla f, f \in \mathcal{F}$. Elements in $\mathcal{L}^{*}$ which are of the form $\nabla f$ are called gradients and $f$ is called the potential of $\nabla f$. Observe that for $f, g \in \mathcal{F}$ elements of the form $g \nabla f$ are in general not gradients. Therefore the gradients do not form an $\mathcal{F}$-module.

An important observation is that the derivative $L_{K}$ can be extended to all tensors. i.e. to all $\mathcal{F}$-multilinear forms on $\mathcal{L}^{*}$ and $\mathcal{L}$. This extension is obtained by defining first

$$
L_{K} G:=[\kappa, G] \text { for all } G \in \mathcal{L}
$$

and then by the requirement that for $L_{K}$ the product rule liolds for tensor products and for those quantities which come from inserting elements of $\mathcal{L}$ and $\mathcal{L}^{* *}$ into $\mathcal{F}$-multilinear forms. This general extension of $L_{K}$ is again called Lie derivative with respect to $K$.

Recall that $\mathcal{F}$-multilinear forms are maps from $\left(a L^{*}\right)^{r} \cup(c \mathcal{L})^{n}, n, r \subset \mathbb{N}$. into $\mathcal{F}$ which are $\mathcal{F}$ linear in each entr. These multilinear forms are called tensors $(n$-times covariant and $r$-times contravariant). Flements of $\mathcal{L}$ and $\mathcal{L}^{*}$ are special tensors which are l-times contravariant and covariant, respectively. In the following we do not distinguish between an $\mathcal{F}$-linear operator $\Theta: \mathcal{L}^{*} \rightarrow \mathcal{L}$ and the tensor $\tilde{\Theta}: \mathcal{L}^{*} \otimes \mathcal{L}^{*} \rightarrow \mathcal{F}$ given b: $\Theta\left(\gamma_{1}, \gamma_{2}\right):=\left\langle\gamma_{1}, \Theta_{\gamma_{2}}\right\rangle$. In the same way we identify operators $J: \mathcal{L} \rightarrow \mathcal{L}^{*}$ and $\Phi: \mathcal{L}-\dot{L}$ with special tensors whicl are two-times covariant and once co-contravariant, respectivels.

To illustrate the construction of $L_{K}$ we compute Lic derivatives for 1-times covariant tensors and for 2 -times contravariant tensors. First, we compute the Lie derivative for som fixed $\gamma \in \mathcal{C}^{*}$. We consider $\langle\gamma, G\rangle, G \in \mathcal{L}$. The product rule applied to $\langle\gamma, G\rangle$ yieds

$$
\left\langle L_{K}(\gamma), G\right\rangle=L_{K}\langle\gamma, G\rangle-\langle\gamma,[K, G]>\text {. }
$$

i.e. the linear map $L_{K}(\mathcal{F}): \mathcal{L}-\mathcal{F}$ is

$$
L_{K}(\gamma)=L_{K} \cdot \gamma-\gamma \cdot L_{K}
$$

For later use we note that for $f \in \mathcal{F}, K \in \mathcal{L}$ the following holds

$$
L_{(f h)}(\gamma)=f L_{h}(\gamma)+\langle\gamma, h>\nabla f .
$$

As an additional example we take some $\mathcal{F}$-linear operator $\theta: \mathcal{L}^{*} \rightarrow \mathcal{L}$. Its Lie derivative $L_{\kappa}$ we compute again by the product rule applied to $\left\langle\gamma_{1}, \Theta_{\gamma_{2}}\right\rangle$ where $\gamma_{1}, \gamma_{2}$ are arbitrar:
chosen elements in $\mathcal{L}^{*}$. This yields

$$
\left.\left\langle\gamma_{1}, L_{K}(\Theta)_{2}\right\rangle=L_{K}\left\langle\gamma_{1}, \Theta \gamma_{2}\right\rangle-\left\langle L_{K}\left(\gamma_{1}\right), \Theta \gamma_{2}\right\rangle<\gamma_{1}, \Theta L_{K}\left(\gamma_{2}\right)\right\rangle
$$

On the right side, the Lie-derivative of the first term is given by definition of the Lie-module. and the Lie derivatives of the $\gamma$ 's were already determined by (4.8). Since $\gamma_{1}$ and $\gamma_{2}$ were 


\section{Hamiltonian Structure and Integrability}

arbitrary, (4.10) defines completely the Lie derivatives for the two-times contravariant tensor $\theta$. In the same way we can define, by induction, the derivative $L_{K}$ for arbitrary tensors.

For purely covariant tensors $\alpha$, i.e. multilinear forms on $(\otimes \mathcal{L})^{n}$ we can define a so called exterior derivative $d$, a notion which plays an important role for hamiltonian vector fields. On $\mathcal{F}$ we define this exterior derivative $d$ to be the gradient

$$
d_{\mid \mathcal{F}}=\Gamma,
$$

and when a tensor is $r$-times covariant $(r \geq 1)$ then we define this exterior derivative by

$$
(d \alpha) \bullet K:=L_{K}(\alpha)-d(\alpha \bullet K) \text { for all } K \in \mathcal{L} \text {. }
$$

Here $\alpha \bullet K$ means that $K$ is inserted as the first entry in the form $\alpha$, for example $\gamma \bullet K=$ $\left\langle, K>\right.$ when $\gamma \in L^{*}$. One easily sees that $(4.12)$ is an inductive definition over the order of covariance. In the following notation we use the convention that $d$ and $L_{K}$ are more binding than $\bullet$, i.e. $d \alpha \bullet K=(d a) \bullet K \neq d(\alpha \bullet K)$, and similarly for the Lie derivative. furthermore we observe that we may use $(+.12)$ as the definition for the exterior derivative also in case of zero-forms $f \in \mathcal{F}$ if we adopt the formal notation that for zero-forms $f$ the expression $f \cdot h$ is equal to zero. More generally, for $n$-forms ( $n$-times covariant tensors) $a$ we define $a \bullet h_{1} \bullet K_{n+1}$ (i.e. application of $n+1 \bullet$ 's) to be zero. This notation will considerably shorten subsequent proofs.

Observation 4.2:

(i) Exterior derivative and Lie-derivative commute.

$$
L_{K} d=d L_{K}
$$

(ii) As usual we obtain that $d \cdot d=0$, a fact which is well known for concrete situations from differential geometry.

Proof:

(i): Consider arbitrary $G, K \in \mathcal{L}$ and covariant $\alpha$, then by use of (4.12) we obtain:

$$
\begin{aligned}
& \left(\left(L_{G} d-d L_{G}\right) \alpha\right) \bullet K-L_{G}(d \alpha \bullet K)-d \alpha \bullet L_{G} K-L_{K} L_{G} \alpha+d\left(L_{G} \alpha \bullet K\right)
\end{aligned}
$$

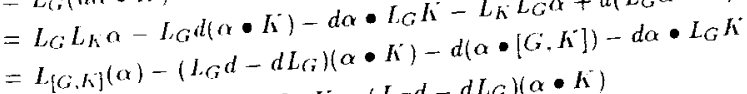

$$
\begin{aligned}
& =d \alpha \cdot[G, K]-(d \alpha) \cdot L_{G} K-\left(L_{G} d-d L_{G}\right)(\alpha \cdot K) \\
& =\left(d L_{G}-L_{G} d\right)(\alpha \cdot K)
\end{aligned}
$$

Hence $\left(\left(L_{G} d-d L_{G}\right) \alpha\right) \bullet K=\left(L_{G} d-d L_{G}\right)(\alpha \bullet K)$ and the claim follows by induction over the order of covariance. Observe that the necessary beginning of our induction argument is given by the fact that - applied to zero-forms gives zero. 
(ii): We again use a repeated argument over the order of covariance. Consider an arbitrary covariant $\alpha$ and arbitrary vertor fields $k, G$, then:

$$
\begin{aligned}
(d \cdot d \alpha) \bullet K \bullet G= & \left(L_{K} d \alpha \bullet G-d(d \alpha \bullet K) \bullet G\right. \\
= & L_{K}(d \alpha \bullet G)-d \alpha \bullet L_{K} G-d\left(d \alpha \bullet K^{*}\right) \bullet G \\
= & L_{K}(d \alpha \bullet G)-d \alpha \bullet L_{K} G-L_{G}(d \alpha \bullet K) \bullet G+d(d \alpha \bullet K \bullet G) \\
= & L_{K} L_{G} \alpha-L_{K} d(\alpha \bullet G)+I_{\{G, K]} \alpha+d(\alpha \bullet[K, G])-L_{G} L_{K} \alpha \\
& \quad \quad+L_{G} d(\alpha \bullet K)+d\left(L_{K} \alpha \bullet G\right)-d(d(\alpha \bullet K) \bullet G) \\
= & d\left(-L_{K}(\alpha \bullet G)-\alpha \bullet\left[G, K^{*}\right]+L_{G}(\alpha \bullet K)+\left(L_{K} \alpha\right) \bullet G-d(\alpha \bullet K) \bullet G\right) \\
= & d\left(L_{G}(\alpha \bullet K)-d(\alpha \bullet K) \bullet G\right) \\
= & d^{2}(\alpha \bullet K \bullet G)
\end{aligned}
$$

Again, the beginning of our induction argument is given by the fact that $\bullet$ applied to zeru-forms gives zero.

\section{Definition 4.3:}

(i) A tensor $T$ is said to be invariant with respect to the vector field $K$ if $L_{K} T=0$.

(ii) Observe that condition (4.5) for $\gamma$ being closed can be written in terms of the exterior derivative as $d \gamma=0$. Thenefore we define a covariant tensor $\alpha$ to bc closed if $d \alpha=0$.

\section{Remark 4.4:}

(i) Gradients are closed bccuuse of $d \cdot d=0$. If $\mathcal{L}$ is the vector field Lie algebra on some manifold $M$ and $\mathcal{F}$ are the scalar fields, then locally the converse is also true (Poincart
Lemmal ( $(25))$.

(ii) Observe that (4.12) implies that any closed covariant tensor $\alpha$ is invariant with respect to $h$ if and only if $\alpha \cdot K$ is again closed. (iii) Let $J$ be some invertible $\mathcal{F}$-linear operator $J: \mathcal{L} \rightarrow \mathcal{L}^{*}$. If $L_{G} J-0$ for all those (
with closed $J G$ then I itself must be closed.

Proof of (ii) and (iii):

(ii): Direct consequence of $(4.12)$.

(iii): Because $\mathcal{L}^{*}$ is generated by its closed elements and since $J$ is invertible we have that $L_{(f K)} J-d(J f K)=f\left(L_{K} J-d(J K)\right.$. vanishes for the subset of those $G$ such that the right side of (4.12) vanishes. 
Now, let $\Theta: \mathcal{L}^{*} \rightarrow \mathcal{L}$ be some antisymmetric $\mathcal{F}$-linear map. We define a bracket in $\mathcal{L}^{*}$ in the following way:

$$
\left\{\gamma_{1}, \gamma_{2}\right\}_{\Theta}:=L_{\left(\Theta \gamma_{1}\right)} \gamma_{2}-L_{\left(\Theta \gamma_{2}\right)} \gamma_{1}+d<\gamma_{1}, \Theta \gamma_{2}>
$$

for $\gamma_{1}, \gamma_{2} \in \mathcal{L}^{*}$.

Before presenting the basic result for these brackets we gather some useful identities. lising (4.12) we can rewrite $(4.14)$ as

$$
\left\{\gamma_{1}, \gamma_{2}\right\}_{\Theta}:=L_{\left(\Theta \gamma_{1}\right)} \gamma_{2}-\left(d \gamma_{1}\right) \bullet\left(\Theta \gamma_{2}\right)
$$

So when $\gamma_{1}$ is closed then by application of (4.12) this bracket reduces to

$$
\left\{\gamma_{1}, \gamma_{2}\right\}_{\Theta}:=L_{\left(\Theta \gamma_{1}\right)} \gamma_{2} \text { for closed } \gamma_{1} \in \mathcal{L}^{*}
$$

Furthermore we easily find with (4.9) how this bracket acts on multiplication with elements of $\mathcal{F}$

$$
\left\{\gamma_{1}, f \gamma_{2}\right\}_{\Theta}:=f\left\{\gamma_{1}, \gamma_{2}\right\}_{\Theta}+\left(L_{\left(\Theta \gamma_{1}\right)} f\right) \gamma_{2} .
$$

Theorem 4.5: Let $\Theta: \mathcal{L}^{*} \rightarrow \mathcal{L}$ be $\mathcal{F}$-linear and antisymmetric, then the following are Equivalent:

(i) $\{,\}_{\Theta}$ defines a Lie algebra among the closed elements of $\mathcal{L}^{*}$

(ii) $\Theta\left\{\gamma_{1}, \gamma_{2}\right\}_{\Theta}=\left[\Theta d \gamma_{1}, \Theta d \gamma_{2}\right]$ for all closed $\gamma_{1}, \gamma_{2} \in \mathcal{L}^{*}$.

(iii) $\{,\}_{\Theta}$ defines a Lie algebra in $\mathcal{L}^{*}$

(iv) $\Theta\left\{\gamma_{1}, \gamma_{2}\right\}_{\Theta}=\left[\Theta \gamma_{1}, \Theta \gamma_{2}\right]$ for all $\gamma_{1}, \gamma_{2} \in \mathcal{L}^{*}$

(x) $L_{\Theta \gamma}(\Theta)=0$ for all closcd $\gamma \in L^{*}$.

(vi) For arbitrary $\gamma \in \mathcal{L}^{*}$ we have that $\Theta$ is invariant with respect to $\Theta \gamma$ if and only if $d \gamma \bullet\left(\Theta \gamma_{1}\right) \bullet\left(\Theta \gamma_{2}\right)=0$ for all $\gamma_{1}, \gamma_{2} \in \mathcal{L}^{*}$.

Proof:

In the following we unit. for simplicity, the sub- $\Theta$ in the brackets \{\}$_{\Theta}$. (i) $\Leftrightarrow$ (ii): Observe that by use of (4.14) then the antisymmetry of $\Theta$ implies for closed $\gamma$ 's
that

$$
\left\{\gamma_{1}, \gamma_{2}\right\}=L_{\left(\Theta d \gamma_{1}\right)} \gamma_{2}=-L_{\left(\Theta d \gamma_{2}\right)} \gamma_{1}
$$

So. $\{$,$\} is antisymmetric anyway, and only the Jacobi identity has to be proved in order$ to show that this is a Lie algebra. Using (4.18) we find for the triple bracket

$$
\begin{aligned}
\left\{\gamma_{3},\left\{\gamma_{1}, \gamma_{2}\right\}\right\} & =-L_{\Theta\left\{\gamma_{1}, \gamma_{2}\right\}} \gamma_{3} \\
& =L_{\left(\Theta \gamma_{3}\right)} L_{\left(\Theta \gamma_{1}\right)} \gamma_{2}=-L_{\left(\Theta \gamma_{3}\right)} L_{\left(\Theta \gamma_{2}\right)} \gamma_{1} .
\end{aligned}
$$


Now, using for the cyclic sum suitable representations obtained from this formula we find with (4.2)

$$
\begin{aligned}
\left\{\gamma_{3}\left\{\gamma_{1}, \gamma_{2}\right\}\right\}+\text { cyclic } & =L_{\left(\Theta d\left\{\gamma_{1}, \gamma_{2}\right\}\right) \gamma_{3}}+L_{\left(\Theta d \gamma_{2}\right)} L_{\left(\Theta d \gamma_{1}\right)} \gamma_{3}-L_{\left(\Theta d \gamma_{2}\right)} L_{\left(\Theta d \gamma_{1}\right)} \gamma_{3} \\
& =\left\{L_{\left(\Theta\left\{\gamma_{1}, \gamma_{2}\right\}\right)}-\left[L_{\left(\Theta d \gamma_{1}\right)}, L_{\left(\Theta d \gamma_{2}\right)}\right]\right\} \gamma_{3} \\
& =\left\{L_{\left(\Theta\left\{\gamma_{1}, \gamma_{2}\right\}\right)}-L_{\left[\Theta d \gamma_{1}, \Theta d \gamma_{2}\right]}\right\} \gamma_{3}
\end{aligned}
$$

Since $\gamma_{3}$ was arbitrary, the $\mathbf{J}$ acobi identity for the triple product can only hold if

$$
L_{\left(\Theta d\left\{\gamma_{1}, \gamma_{2}\right\}\right)}-L_{\left[\Theta d \gamma_{1}, \Theta \gamma_{2}\right]}=0
$$

which is equivalent to (ii) since $K \rightarrow L_{K}$ was assumed to be injective. On the other hand, whenever (ii) (and (4.20) as a consequence) holds then the cyclic sum obviously must be equal to zero, which implies the Jacobi identity.

The implications (iv) $\rightarrow$ (ii) and (iii) - (i) are obvious.

(ii) - (iv) and (i) - (iii) are either done by direct computation or by using remark 4.1 together with (4.17). To see this observe that (i) gives a Lie algebra $\left(\mathcal{L}_{c l}^{*},\{\}\right)$, where $\mathcal{L}_{c l}^{*}$ are the closed elements in $\mathcal{L}^{*}$ and that (4.17) gives a Lie algebra homomorphism $\gamma \rightarrow L_{\gamma}^{*}=L_{\theta \gamma}$ from $\mathcal{L}_{d}^{*}$ into the derivations of $\mathcal{F}$. Now taking the unique extension (described in remark 1.1) to the $\mathcal{F}$-module generated by $\mathcal{L}_{r l}^{*}$ (which by definition is equal to $\mathcal{L}^{*}$ ), one obtains the Lie algebra defined by (1.14). The fact that the lomomorphism $\Theta$ extends from $\mathcal{L}_{d l}^{*}$ to $\mathcal{L}^{*}$ is due to the property that $\Theta L_{\gamma}^{*}=L_{\Theta \gamma}$.

(v) $\Leftrightarrow$ (ii): By (4.16) the condition

$$
\Theta L_{(\Theta \gamma)} \gamma_{1}=L_{(\Theta \gamma)}\left(\Theta \gamma_{1}\right) \text { for all closed } \gamma, \gamma_{1} \in \mathcal{L}^{*}
$$

is equivalent to (ii). And by the product rule this is equivalent to (v).

(vi) $\Leftrightarrow$ (iv): Using (4.15) and the antisymmetry of $\Theta$ we find for arbitrary $\gamma, \gamma_{1}, \gamma_{2} \in \mathcal{L}^{*}$

$$
\begin{aligned}
\left\langle\gamma_{1},\left(L_{\left(\Theta_{\gamma}\right)} \Theta\right) \gamma_{2}\right\rangle & =\left\langle\gamma_{1}, L_{(\Theta-)}\left(\Theta \gamma_{2}\right)>-\left\langle\gamma_{1}, \Theta L_{(\Theta \gamma)} \gamma_{2}>\right.\right. \\
& =\left\langle\gamma_{1},\left[\Theta \gamma, \Theta \gamma_{2}\right]>-\left\langle\gamma_{1}, \Theta\left\{\gamma, \gamma_{2}\right\}>+\left\langle\gamma_{1}, \Theta\left(d \gamma \bullet \Theta \gamma_{2}\right)\right\rangle\right.\right. \\
& =\left\langle\gamma_{1},\left[\Theta \gamma, \Theta \gamma_{2}\right]>-\left\langle\gamma_{1}, \Theta\left\{\gamma, \gamma_{2}\right\}>-d \gamma_{1} \bullet\left(\Theta \gamma_{2}\right) \bullet\left(\Theta \gamma_{1}\right) .\right.\right.
\end{aligned}
$$

Hence we obtain

$$
<\gamma_{1},\left(L_{\Theta-} \Theta \eta_{2}>+d \gamma_{1} \bullet\left(\Theta \gamma_{2}\right) \bullet\left(\Theta \gamma_{1}\right)=<\gamma_{1},\left[\Theta \gamma, \Theta \gamma_{2}\right]-\Theta\left\{\gamma, \gamma_{2}\right\}>\right.
$$
Equating the right side of $(4.22)$ to zero is equivalent to (iv) and equating its left side to
zero is equivalent to $(v)$.

Let us have a closer look at condition ( $v)$ : If $\Theta$ is invertible, then the condition therin imposed on $\gamma$ is equivalent to $d \gamma=0$, i.e. $\gamma$ must be closed. Hence, for $J:=\Theta^{-1}$ condiat 4.4 (ii) and (iii) we see that for an invertible $\Theta$ one of the equivalent conditions of the theorem above is fulfilled if and only if $J=\Theta^{-1}$ is closed. In the finite dimensional theory 
the antisymmetric closed invertible $J$ are called symplectic. So, loosely speaking, $\Theta$ has the algebraic behaviour of the inverse of a symplectic operator. Therefore, if one of the conditions of Theorem 4.5 is fulfilled, $\Theta$ is said to be implectic, a name which stands for inverse symplectic. Sometimes instead of implectic, the name Poisson tensor is chosen. For reasons which will become obvious in the next section the $\{,\}_{\Theta}$ are called the Poisson brackets with respect to $\Theta$.

We decided not to insist on the invertibility condition because of the infinite dimensional nature of our manifolds, so we have to extend the notion symplectic for this more general situation: An operator $J: \mathcal{L} \rightarrow \mathcal{L}^{*}$ is called symplectic if it is antisymmetric and closed, and if in addition its kernel $\operatorname{ker}(J)=\{G \in \mathcal{L} \mid J G=O\}$ is a Lie ideal in $\mathcal{L}$. Being a Lie ideal means of course that $[K, G] \in \operatorname{ker}(J)$ for all $G \in \operatorname{ker}(J)$ and $K \in \mathcal{L}$. This additional ideal-condition is automatically fulfilled if $J$ is invertible because then $k \cdot e r(J)=0$.

In a analogy to implectic operators one can use symplectic operators $J$ to construct in $J \mathcal{L}$ suitable Poisson brackets: Take $\gamma_{1}, \gamma_{2} \in J \mathcal{L}$ and choose $G_{1}, G_{2}$ such that $\gamma_{i}=J G_{i}, i=$ 1.2. Then we define

$$
\left\{\gamma_{1}, \gamma_{2}\right\}^{(J)}:=L_{G_{1}}\left(\gamma_{2}\right)-L_{G_{2}}\left(\gamma_{1}\right)+\left\langle\gamma_{1}, G_{2}>\right.
$$

Rewriting that with (4.12) and using $d(J)=0$ we obtain

$$
\left\{\gamma_{1}, \gamma_{2}\right\}^{(J)}=L_{G_{1}}\left(G_{2}\right)-d\left(J G_{1}\right) \bullet G_{2}=J L_{G_{1}} G_{2}+d(J) \bullet G_{1} \bullet G_{2}=J\left[G_{1}, G_{2}\right] .
$$

Since $\operatorname{ker}(J)$ is all ideal with respect to $[$,$] the bracket \{,\}^{(J)}$ does not depend on the choice of $G_{1}, G_{2}$. Furthermore, because $J$ is one-to-one from the equivalence classes modulo $\operatorname{ker}(J)$ to $J \mathcal{L}$, the bracket $\{.\}^{(J)}$ must be a Lie algebra such that $J$ is a homomorphism from $\{,\}^{(J)}$ into $[$,

We may summarize this section: An implectic operator makes out of $\mathcal{L}^{*}$ a Lie algebra module $\left(\mathcal{L}^{*},\{,\}_{\Theta}, \mathcal{F}\right)$, with corresponding $\mathcal{F}$-derivations $L_{\gamma}^{*}, \gamma \in \mathcal{L}^{*}$ such that $\Theta$ is a homomorphism from this Lie algfbra module into $(\mathcal{L},[],, \mathcal{F})$. Here homomorphism means that for all $\gamma_{1}, \gamma_{2} \in \mathcal{L}^{*}$ we have

$$
\Theta\left\{\gamma_{1}, \gamma_{2}\right\}_{\Theta}=\left[\Theta \gamma_{1}, \Theta \gamma_{2}\right]
$$

and

$$
\Theta L_{\gamma_{1}}=L_{\Theta \gamma_{1}} \text {. }
$$

In general, it is highly desirable to construct for given tensors suitable Lie algebra elements which leave these tensors invariant. Indeed, as we will see, the search for symmetries, conservation laws, invariant spectral problems and the like may be subsumed under this general theme. In view of that problem it is obvious that symplectic and implectic tensors must play a fundamental role because for them the closed elcments in $\mathcal{L}^{*}$ imediatly give rise to such invariances. 


\section{$5 \quad$ Hamiltonian and Bi-Hamiltonian Fields}

In this section we always assume that $\Theta$ is an implectic operator. For some of the results presented here the reader is referred to the fundamental papers $([10],[11],[12],[14],[15)]$.

Comparing the different Poisson brackets defined in Sections 3 and 4, one discovers that they are defined on rather different spaces. However, these two notions are easily connected if, as before in the concrete situation, a bracket among the elements of $\mathcal{F}$ is
defined as follows:

$$
\left\{f_{1}, f_{2}\right\}_{\Theta}:=<d f_{2}, \Theta d f_{1}>=L_{\left(\Theta d f_{1}\right)} f_{2} \text { for } f_{1}, f_{2} \in \mathcal{F} .
$$

One easily sees that $\nabla$ maps the $\mathcal{F}$-brackets into the $\mathcal{L}^{*}$-brackets:

$$
\nabla\left\{f_{1}, f_{2}\right\}_{\Theta}=\left\{\nabla f_{1}, \nabla f_{2}\right\}_{\Theta} .
$$

This suggests that these $\mathcal{F}$-brackets also form a Lie algebra. Indeed this is the case, the proof is literally almost the same as the proof (i) $\Leftrightarrow$ (ii) in the last theorem ${ }^{7}$.

\section{Definition 5.1:}

(i) Elements $i \in \mathcal{L}$ which are of the form $K=\Theta \gamma$ with closed $\gamma$ and inplectic $\Theta$ art called hamiltonian (with respert to $\Theta$ ).

(ii) Given some symplectic $J$, then elements $K \in \mathcal{L}$ such that $J K$ is closed are called inverse-hamiltonian ${ }^{8}$ (with respect to $J$ ).

(iii) Let $\Theta$ be implectic and $J$ be closed, and assume that $J$ is not the inverse of $\Theta$. Then some $K$ is called a bi-hamiltonian field (with respect to $\Theta$ and $J$ ) if $J K$ is closed
and if there is some closed $\gamma$ such that $K=\Theta \gamma$.

The power of bi-hamiltonian fields is seen from:

Observation 5.2: Consider a bi-hamiltonian field

as described aboce, and define $K_{n+1}=(\Theta J)^{n} K$. Then all the $K_{n}$ and $\gamma_{n}:=J K_{n}$ are
invariant with respect to $K$.

$$
\Pi=\Theta_{\gamma}, J \Lambda=\gamma_{1}, \gamma \text { and } \gamma_{1} \text { being closed }
$$

Proof:

\footnotetext{
An interesting question is whether or not

guarantee that $\Theta$ is implectic whether or not the Jacobi identity for the $\mathcal{F}$. In

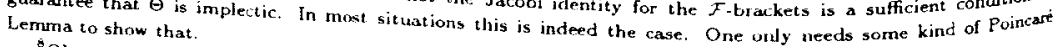
${ }^{\circ}$ Observe that for

reason why in the fonite dimensional or $J$ the nuciuns hamittonian and inverse-hamiltonian do coincide. This is the forms are usually assumed to be non-degen the notion inverse-hamiltonian does not appear, since there symplectic nondegenerary is not advised because invertibility. In the infinite dimensional situation however, the assumption of very much on the spaces under consideratibility of operators usually involves topological considerations and depends
} 
lising the bi-hamiltonian nature of $K$ we see from Remark 4.4 (ii) and from Theorem 4.5 (v) that $\Theta$ and $J$ are invariant with respect to $K$. Hence, by the product rule, all $K_{n}$ have to be invariant with respect to $K$.

Let us now illustrate the notions and techniques of the last chapter in the light of the concrete situation which was considered in Sections 2 and 3.

\section{Standard situation :}

Let $M$ be a $C^{\infty}$-manifold, we consider $C^{\infty}$-tensor fields on $M$. In particular let $\mathcal{L}$ be the vector fields, $\mathcal{F}$ be the scalar fields and let $\mathcal{L}^{*}$ be those $C^{\infty}$-maps from $M$ into the cotangentbundle which are given by assigning to $u \in M$ a continuous linear functional on the tangent space $T_{u} M$ at $u$.

In order to carry out computations with Lie derivatives we should show what these look like in charts. As we already know, for a scalar field $f$ the Lie derivative is the usual gradient

$$
L_{A} f=\langle\nabla f, A\rangle=f^{\prime}[A]
$$

and the application of $L_{A}$ to a co-vector field $\gamma$ is found to be

$$
L_{A} \gamma=\gamma^{\prime}[A]+A^{\prime+} \gamma
$$

where $A^{\prime+}$ denotes the transpose of the operator $A^{\prime}$ with respect to the duality between tangent and cotangent space.

Furthermore, if $\Theta: T^{*} M \rightarrow T M$ and $J: T M \rightarrow T^{*} M$ are two-times contravariant and two-times covariant, respectively, then their Lie derivatives are

$$
\begin{aligned}
L_{A} \Theta & =\Theta^{\prime}[A]-\Theta A^{\prime+}-A^{\prime} \Theta \\
L_{A} J & =J^{\prime}[A]+A^{\prime+} J+J A^{\prime}
\end{aligned}
$$

Finally the Lie derivative for an operator $\Phi: T M \rightarrow T M$ is equal to

$$
L_{A} \Phi=\Phi^{\prime}[A]-A^{\prime} \Phi+\Phi A^{\prime} \text {. }
$$

Fix some $K \in \mathcal{L}$, then $f \in \mathcal{F}$ and $G \in \mathcal{L}$ are invariant with respect to $K$ if and only if $f$ is a conserved quantity and $G$ a symmetry group generator, respectively. Using the Lie derivatives, we that $\Theta$ is implectic if and only if condition 3 (ii) of Theorem 3.2 is fulflled. In the same way we obtain as equivalent condition for $J$ being closed that

$$
\left.<J(u)^{\prime}\left[G_{1}\right] G_{2}, G_{3}>+<J(u)^{\prime}\left[G_{2}\right] G_{3}, G_{1}\right\rangle+\left\langle J(u)^{\prime}\left[G_{3}\right] G_{1}, G_{2}>=0\right.
$$
must hold for all $G_{1}, G_{2}, G_{3} \in \mathcal{L}$. Hence Section 4 generalizes the situation considered in
Section 3 .

Now, we consider again the dynamical system

$$
u_{t}=K(u) \text {. }
$$


In analogy to Definition 5.1 we call (1.1) a bi-hamiltonian system if there are closed $J(u)$, implectic $\Theta(u)$ and closed $\gamma_{0}, \gamma_{1}$ such that

$$
K=\theta \gamma_{0} \text { and } J K=\gamma_{1}
$$

If that is the case then we obtain as consequence of 5.2

Observation 5.3: Define induclively

$$
\begin{gathered}
K_{1}=K \text { and } K_{n+1}=\theta J K_{n} \\
\gamma_{n+1}=J \Theta \gamma_{n}
\end{gathered}
$$

then all the $K_{n}$ and $\gamma_{n}$ are invariant with respect to $K$. Hence the $K_{n}$ are symmetry group generators for (1.1) and, if the $\gamma_{n}$ are gradients, then they are gradieruls of conserved quantities for the evolution equation (1.1).

\section{Example 5.4: Korteweg-de Vries equation}

Consider the situation as in Example 3.5 . We observe that the inverse of $D$

$$
\left(D^{-1} f\right)(x):=\int_{-\infty}^{x} f(\xi) d \xi
$$

is a well defined operator $D^{-1}: \mathcal{S} \rightarrow \mathcal{S}^{*}$. The Korteweg-de Vries equation

$$
u_{t}=H(u):=6 u u_{x}+u_{x x x}
$$

is a bi-hamiltonian system since for the implectic $\Theta=D^{3}+2(D u+u D)$ and the symplectic $J=D^{-1}$ we have that (5.9) is fulfilled when $\gamma_{0}$ and $\gamma_{1}$ are taken to be the gradients of $I_{0}$ and $I_{1}$ as given in (3.10) and (3.11), respectively. Hence putting

$$
h_{n+1}:=(\Phi)^{n} K \text {, where } \Phi=D^{2}+2 D u D^{-1}+2 u,
$$

then all these $h_{n}$ are symmetry group generators. Since $\Phi$ is recursively generating sym thetry group generators, this usually is called a recursion operator [20(]sometimes also a strong symmetry, for example in $[21,[[5,[3)]$.

mains a major problem is very useful for constructing symmetry groups, there still reconstructing suitab]e coordiuates ans of the system (1.1). Ultimately, we are interested in which are invariant under the flow (action variables) for the flow, so we need scalar quantities generators, however the the flow. Certainly, action variables give rise to symmetry group fields may not be closed. Therefor not always true, hecause the corresponding co-vector of great importance. If that hapefore the question whether or not the fields $\gamma_{n}$ are closed is construct suitable coontinat hapens then, by the Poincare lemma, one can, at least locally. is a vector space thendinates. And if, as in the example, the manifold under consideration al potentials is a simple excercise:

Lemma 5.5: Let the manifold of all $u$ 's be a vector space. Then a co-vector field $\gamma^{(u)}$ Proof: 
Since gradients are closed we only have to prove the existence of $F$ for closed $\gamma$. So let $\gamma(u)$ be closed. Consider the scalar field

$$
F(u)=\int_{0}^{1}<\gamma(\lambda u), u>d \lambda
$$

Take some arbitrary $v(u)$ in the tangent bundle. Then by $(5.4)$ we obtain

$$
\begin{aligned}
\langle\nabla F(u), v\rangle & =\int_{0}^{1}\left\{\lambda\left\langle\gamma^{\prime}(\lambda u)[v], u\right\rangle+\langle\gamma(\lambda u), v\rangle\right\} d \lambda \\
& =\int_{0}^{1}\left\{\left\langle\lambda \gamma^{\prime}(\lambda u)[u], v\right\rangle+\langle\gamma(\lambda u), v\rangle\right\} d \lambda \\
& =\int_{0}^{1} \frac{d}{d \lambda}\{\langle\lambda \gamma(\lambda u), v\rangle\} d \lambda=\langle\gamma(u), v\rangle
\end{aligned}
$$

This proves $\nabla F(u)=\gamma(u)$ since $u(u)$ was arbitrary

Observe that this proof can be generalized to the situation where the manifold can be parametrized by a star-shaped subset of a vector space.

\section{Compatibility}

In this section we introduce the essential structure which will be responsible for the fact that in most cases of bi-hamiltonian systems the invariant co-vector fields constructed by use of Observation 5.2 are indeed closed. Again we will consider the necessary arguments at a rather general level. This is not done in order to introduce an unreasonable level of abstraction but rather to get rid of unnecessary ballast. Further, it turns out that with a general formulation of compatibility in Lie algebras one is more flexible with respect to applications. In our presentation we follow closely [9.]

Let, $(\mathcal{L},[]$,$) be some Lie algebra over \mathbb{R}$ or $(\mathbb{C}$. We call $(\mathcal{L},[]$,$) the reference$ algebra. Let furthermore $\Lambda$ be a vector space over the same scalars. Now, consider a linear transformation

$$
T: \Lambda \rightarrow \mathcal{L}
$$

from $\Lambda$ into the reference algebra $\mathcal{L}$. We call some (bilinear) product $\{$, $\}$ in $A$ (not necessarily assumed to be a lie product) a $T$-product if $T$ is a homomorphism into $(\mathcal{L},[]$,$) ,$ i.e. if

$$
T\{a, b\}=[T a, T b] \text { for all } a, b \in \Lambda .
$$

To emphasize that some product is a $T$-product we write $[]$,$T instead of \{\}$. A linear $\Phi: \mathcal{L} \rightarrow \mathcal{L}$ is said to be hereditary if

$$
[a, b]_{\Phi}:=[\Phi a, b]+[a, \Phi b]-\Phi[a, b]
$$
defines a $\Phi$-product in $\mathcal{L}$. Using then $\Phi[a, b] \Phi=[\Phi a, \Phi b]$, we see that $\Phi$ is hereditary if and
only if:

$$
\Phi^{2}[a, b]+[\Phi a, \Phi b]=\Phi\{[\Phi a, b]+[a, \Phi b]\} \text { for all } a, b \in \mathcal{L}
$$



Rephrasing a notion introduced earlier we call a linear map $\Phi: \mathcal{L} \rightarrow \mathcal{L}$ invariant witt
respect to $k \in \mathcal{L}$ if

$$
\Phi[k, b]=[k, \Phi b] \text { for all } b \in \mathcal{L}
$$

Theorem 6.1: Let $\Phi$ be a hereditary map which is invariant with respect to $k$. Then $\left\{\Phi^{n} k \mid n \in N_{0}\right\}$ is an abelian subset of $\left(\mathcal{L},[\mid)\right.$. If $\Phi$ is invertible then $\left\{\Phi^{n} k \mid n \in \mathbb{Z}\right\}$ is
abelian as uell. For the proof we need:

Lemma 6.2: Let $\Phi$ be hereditary and let it be invariant with respect to $k$. Then $\Phi$ is invariant with respect to $\Phi k$. If $\Phi$ is invertible then it is invariant with respect to $\Phi^{-1} k$ as
well. Thus the set well. Thus the set $\{k \mid \Phi$ invariant with respect to $k\}$ of all elements which leave $\Phi$ invari. ant is a subalgebra of $\mathcal{L}$ which is invariant under the application of $\Phi$ (and of $\Phi^{-1}$ if $\Phi$ is
invertible).

Proof: Replace $a$ by $k$ in $(6.3)$. Since $\Phi$ is invariant with respect to $k$ the first and fourth term
cancel and the equality reads

$$
[\Phi k, \Phi l]=\Phi[\Phi k, b] \text { for all } b
$$

This clearly implies that $\Phi$ is invariant with respect to $\Phi k$. If $\Phi$ is invertible, we replace $a$ in (6.3) by $\Phi^{-1} k$ and apply $\Phi^{-1}$ to the remaining two terms.

Proof of Theorem 6.1: From the Lemma 6.2 we obtain by induction that $\Phi$ is invariant with respect to any $\Phi^{m} k$
and $\Phi^{n} k$. Hence using antisymmetry we find

$$
\left[\Phi^{m} k, \Phi^{n} k\right]=\Phi^{m+n}[k, k]=0
$$

for all $m, n$. For invertible $\Phi$, in this argument $\Phi^{-1}$ has to replace $\Phi$

Remark 6.3: Let $\Phi$ be hereditary and let $a_{1}$ and $a_{2}$ be eigenvectors of $\Phi$, i.e.

Then for these $a_{i}$ relation (6.3) is equivalent to

$$
\Phi a_{i}=\lambda_{i} a_{i} . \lambda_{i}=\text { scalar, } i=1,2 .
$$

$$
\left(\Phi-\lambda_{1}\right)\left(\Phi-\lambda_{2}\right)\left[a_{1}, a_{2}\right]=0 .
$$

Hene, when an operator $\Phi$ has a spectral resolution, this operator is hereditary if and only if all the corresponding spectral projections are algebra honomorphisms.

have $T$ - and $\Psi$-products $l$ situation of maps from $\Lambda$ into $\mathcal{L}$. Assume that in $A$ we compatible if

defines a $(T+\Psi)$-product in $\Lambda$.

$$
\{a, b\}:=[a, b]_{T}+[a, b]_{\psi}
$$

Lemma 6.4: Let $[\text {, }]_{T}$ and $[,]_{\Psi}$ be $T$ - and $\Psi$-products, respectively. These products

$$
T[a, b]_{\Psi}+\Psi[a, b]_{T}=[T a, \Psi b]+[\Psi a, T b] \text { for all } a, b \in \Lambda
$$


Proof:

Observe that $T[a, b]_{T}=[T a, T b]$ and $\Psi[a, b]_{\Psi}=[\Psi a, \Psi b]$. So, $(6.8)$ is obviously equivalent to

$$
(T+\Psi)\left\{[a, b]_{T}+[a, b]_{\Psi}\right\}=[(T+\Psi) a,(T+\Psi) b],
$$

which proves the claim.

Observation 6.5: Let $\lambda$ be a scalar. Obviously. [, ] $]_{\lambda T}$ defined by $[a, b]_{\lambda T}:=\lambda[a, b]_{T}$ is a $(\lambda T)$-product whenever $[,]_{T}$ is a $T$-product. Now, replacing in $(6.8) \Psi$ and $[,] \Psi$ by $\lambda \Psi$ and $[,]_{\lambda \Psi}$, respectively, we see that $(6.8)$ remains valid. In other words, $(6.8)$ is linear in $\Psi$ (as well as in $T$ ). Hence, if $[,]_{T_{1}}$ and $[,]_{T_{2}}$ are compatible, and if both are compatible with $[,]_{T}$ then $\left[, X_{T_{1}}+[,]_{\sigma l_{2}}\right.$ is always compatible with $[,]_{T}$.

Observation 6.6: Consider the case when the reference algebra is equal to $\Lambda$, i.e. $\Lambda=\mathcal{L}$ and put $T=I, \Psi=\Phi$. Furthernore, assume that $[,]_{T}$ is the given product in $(\mathcal{L},[])$, and that $[,]_{\Psi}=\{\}$ is a second product such that $\Phi:(\mathcal{L},\{\})-(\mathcal{L},[])$ is a homomorphism. Then (6.8) holds if and only if \{\} is the product defined in (6.3). Hence, $\Phi$ is hereditary if and only if $(\mathcal{L},\{\}$,$) and (\mathcal{L},[]$,$) are compatible.$

In order to shorten our notions we call $\Psi$ and $T$ compatible if their $\Psi$-and $T$-products, $\left[,\left.\right|_{\Psi}\right.$ and $[,]_{T}$ are compatible. By application of this notion to the special case of hered itary operators $\Phi_{1}, \Phi_{2}$ we see that $\Phi_{1}$ and $\Phi_{2}$ are compatible if and only $\Phi_{1}+\Phi_{2}$ is again leereditary.

Theorem 6.7: Consider maps $T, \Psi: \Lambda \rightarrow \mathcal{L}$ and thcir corresponding products $[,]_{T}$ and [. $\Psi$. Assume that $\Psi$ is invertible. Then $T$ and $\Psi$ are compatible if and only if $\Phi=T \Psi^{-1}$ is hereditary.

Proof:

Define a second product in $\mathcal{L}$ by

$$
\{a, b\}:=\Psi\left[\Psi^{-1} a, \Psi^{-1} b\right]_{T} \text { for } a, b \in \mathcal{L}
$$

Then $\Phi:(\mathcal{L},\{\}) \rightarrow(\mathcal{L},[]$,$) is a homomorphism. Using the definition of \{$.$\} and (6.1)$ (for $\Psi$ instead of $T$ ) we obtain

$$
\begin{aligned}
(I+\Phi)([a, b]+\{a, b\}) & =(T+\Psi) \Psi^{-1}([a, b]+\{a, b\}) \\
& =(T+\Psi)\left(\left[\Psi^{-1} a, \Psi^{-1} b\right]_{\Psi}+\left[\Psi^{-1} a, \Psi^{-1} b\right]_{T}\right) .
\end{aligned}
$$

For general $a, b \in L$ the right side is equal to $\left[(T+\Psi) \Psi^{-1} a,(T+\Psi) \Psi^{-1} b\right]$ if and only if $T$ and $\Psi$ are compatible, but this expression is equal to $[(I+\Phi) a,(I+\Phi) b$ and equal to the left side if and only if $I$ and $\Phi$ are compatible. Hence the compatibility of $T$ and $\Psi$ and that of $I$ and $\Phi$ are equivalent. Now using Observation 6.6 we see that this is equivalent to $\Phi$ being hereditary.

By similar arguments we find:

Observation 6.8: Let $\Phi, \Psi$ be compatible hereditary operators and assume that $\Phi$ and $\Phi$ do commutc. Then $\Phi \Psi$ is hereditary. As a consequence. if $\Phi$ be hereditary, then any polynomial in $\Phi$ is hereditary. 
Now consider again the

Lie-module situation:

Let $(\mathcal{L},[], \mathcal{F})$ be a Lie module as considered in Section 4 and define $\Lambda=\mathcal{L}^{*}$. If $\Phi: \mathcal{L} \rightarrow \mathcal{L}$ is a tensor, then condition (6.3) is easily rephrased as

$$
\Phi L_{A}(\Phi)=L_{(\Phi A)}(\Phi) \text { for all } A \in \mathcal{L}
$$

So $\Phi$ is hereditary if and only if (6.10) is fulfilled. In case of the standard situation this car be expressed in charts as

$$
\Phi \Phi^{\prime}[A] B-\Phi\left[\Phi^{\prime} A\right] B=\Phi \Phi^{\prime}[B] A-\Phi\left[\Phi^{\prime} B\right] A \text { for all } A, B \in \mathcal{L}
$$

a condition which appeared in [3.] Because of Theorem 4.5 (4.14) defines a $\Theta$-product in $\mathcal{L}^{*}=\Lambda$ if and only if $\Theta$ is implectic. Since $\Theta$ enters the definition (4.14) linearly we have:

Observation 6.9: Two implectic operators $\Theta_{1}, \Theta_{2}$ are compatible if and only if $\Theta_{1}+\Theta_{\text {. }}$
is again implectic.

From Theorem 6.7 we obtain:

Corollary 6.10: Let $\Theta_{1}, \Theta_{2}$ be implectic and assume that $\Theta_{1}$ is invertible. Then $\Theta_{1}+\theta_{2}$ is implectic if and only if $\Phi=\Theta_{2} \Theta_{1}^{-1}$ is hereditary.

These results we apply to the

Bi-hamiltonian case: Let $\Theta_{1}, \Theta_{2}$ be compatible implectic operators such that $\Theta_{1}$ is in vertible. Let $H^{-}$be a bi-hamiltonian vector field

Then define

$$
\Lambda^{\circ}=\Theta_{1} \gamma_{1}=\Theta_{2} \gamma_{2}, \gamma_{1}, \gamma_{2} \text { closed }
$$

\section{Theorem 6.11:}

$$
\begin{gathered}
\Phi:=\Theta_{2} \Theta_{1}^{-1}, \Phi^{+}:=\Theta_{1}^{-1} \Theta_{2} \\
J_{n}:=\Theta_{1}^{-1} \Phi^{n}, \Theta_{n+1}=\Phi^{n} \Theta_{1}
\end{gathered}
$$

(i) All $J_{n}$ and all $\gamma_{n}$ are closed.

(ii) All tensors $\Phi, \Phi^{+}, K_{n}, \gamma_{n}, J_{n}, \Theta_{n}$ are invariant with respect to every $K_{m}$, in particular
all $K_{n}^{-}, K_{m}^{-}$commute.

(iii) If, in addition, $\Theta_{2}$ is invertible, then the $\Theta_{n}$ are implectic.

Before we can prove this we need to introduce a canonical extension $\overline{\mathcal{L}}$ of $\mathcal{L}$.

The affine extension of $\mathcal{L}$ : 
Let $\tilde{\mathcal{L}}=\mathcal{L} \otimes \mathbb{C}[\xi]$ be the Lie algebra of formal power series in the indeterminate $\xi$ with coefficients in $\mathcal{L}$. Extend in the same way $\mathcal{F}$ and $\mathcal{L}^{*}$

$$
\tilde{\mathcal{F}}=\mathcal{F}: \mathbb{C}[\xi], \hat{L}^{*}=\mathcal{L}^{*} \otimes \mathbb{C}[\xi]
$$

Define that all Lie derivatives, and consequently the exterior derivative, ignore the variable s.i.e.

$$
\left[\sum_{n} A_{n} \xi^{n}, \sum_{m} B_{m} \xi^{m}\right]=\sum_{n, m}\left[A_{n}, B_{m}\right] \xi^{n+m} .
$$

Obviously $(\overline{\mathcal{L}},[], \tilde{\mathcal{F}})$ is again a Lie module. We can embed the tensor structure of $\mathcal{L}$ into that of $\tilde{\mathcal{L}}$ by treating $\xi$ as scalar. By comparison of coefficients we then obtain that a covariant tensor $T[\xi]$ with respect to $\dot{\mathcal{L}}$

$$
T[\xi]=\sum T_{n} \xi^{n}
$$

given by a formal power series in $\xi$ with tensors in $\mathcal{L}$ as coefficients) is closed if and only if all the $T_{n}$ are closed. Now having this additional structure we present as a simple excercise:

Proof of Theorem 6.11:

Consider the affine extension $\dot{\mathcal{L}}$ of $\mathcal{L}$. Since $\Theta_{1}, \Theta_{2}$ are compatible implectic operators we have that $\tilde{\Theta}: \dot{L}^{*}-\overline{\mathcal{L}}$ defined by $\tilde{\Theta}=\Theta_{1}+\xi \Theta_{2}$ is again implectic (Observation 6.5). Observe that $\dot{\mathcal{\Theta}}$ has an inverse $\tilde{J}$

$$
\tilde{J}=\bar{\Theta}_{1}^{-1}=\Theta_{1}^{-1} \sum_{n=0}^{\infty}(-\xi)^{n}\left(\Theta_{2} \Theta_{1}^{-1}\right)^{n} .
$$

Hence $j$ must be closed in $\tilde{L}$. Therefore every

$$
J_{n}=\Theta_{1}^{-1}\left(\Theta_{2} \Theta_{1}^{-1}\right)^{n}=\Theta_{1}^{-1}(\Phi)^{n}
$$

mist be closed in $\mathcal{L}$. This is the essential step where we needed the extension of $\mathcal{L}$. From l:ow on we argue in $\mathcal{L}$.

From the bi-hamiltonian formulation we know (Observation 5.2) that the $\Theta_{1}, \Theta_{2}, \Phi$ and all the $K_{n}$ are $K$-invariant (product rule).

(1): We already know that $J_{n}$ is closed and $K$-invariant. Hence by Remark 4.4 (ii) the $\because=J_{n} I^{\prime}$ must be closed. This holds for all $n$.

(i): We know that $\Phi$ is invariant with respect to all the $K_{m}$ (consequence of Lemma 6.2). since $\Theta_{1}^{-1}$ and the $\gamma_{n}$ are closed, $\Theta_{1}^{-1}$ must be invariant with respect to $K_{n}=\Theta_{1} \gamma_{n}$ (Remark Since $\Theta_{1}^{-1}$ and the $\gamma_{n}$ are closed, $\Theta_{1}^{-1}$ must be invariant with respect $\Theta_{n+1}=\Phi^{m} \Theta_{1}$ are $K_{n}$ -
invariant. By the product rule we then find that $J_{m}=\Theta_{1}^{-1} \Phi^{m i}$ and $\Theta_{m}$

iii): If $\Theta_{2}$ is invertible as well we may interchange the role of $\Theta_{1}$ and $\Theta_{2}$ in order to see that $j_{n}=\Theta_{2}^{-1} \Phi^{-n}$ is closed. So its inverse $\Theta_{n}^{-1}$ must be implectic.

\section{Examples and Applications}

Observe that when the duality between tangent and co-tangent space is represented as in Example 3.5 then the differential operator $D$ is trivially implectic. In this section we show how, from this knowledge, new pairs of implectic operators can be constructed. 
We first point out the relation between Lei-module-isomorphisms and variable transformations. This connection allows efficient use of the invariant manner in which we intro-
duced the main notions.

Consider Lic modules $(\mathcal{L}, \mathcal{F})$ and $(\tilde{\mathcal{L}}, \tilde{\mathcal{F}})$. In both modules we denote the Lie product by [] since no confusion arises. A pair $(S, \sigma)$ of maps $S: \mathcal{L} \rightarrow \tilde{\mathcal{L}}$ and $\sigma: \mathcal{F} \rightarrow \tilde{\mathcal{F}}$ is said to
be a Lie-module-homomorphism if

- $S$ and $\sigma$ are homomorphisms with respect to the algebraic structures in $\mathcal{L}$ and $\mathcal{F}$
respectively,

- $S\left(f K^{\circ}\right)=\sigma(f) S(K)$ for all $f \in \mathcal{F}$ and $K \in \mathcal{L}$.

- $S \cdot L_{K}=L_{(S(K))}$ for all $K \in \mathcal{L}$.

If $S$ and $\sigma$ are invertible then this is called a Lie-module-isomorphism. Isomorphisms define for $\gamma \in \mathcal{L}^{*}$ the the whole tensor structure from $(\mathcal{L}, \mathcal{F})$ to $(\overline{\mathcal{L}}, \overline{\mathcal{F}})$. To do this we first define for $\gamma \in \mathcal{L}^{* *}$ the corresponding $\tilde{\gamma} \in \tilde{\mathcal{L}}^{*}$ by

$$
\left\langle\bar{\gamma}, \bar{K}>:=\sigma\left(<\gamma, S^{-1} \bar{K}>\right)\right. \text {. }
$$

The map $S^{*}: \gamma-\bar{\gamma}$ is called the reciprocal image. Let $\Psi$ be some $\mathcal{L}$-tensor $(r$-times contravariant and $n$-times covariant), then the corresponding $\tilde{\mathcal{L}}$-tensor $\tilde{\Psi}$ is defined by $\left.\Psi_{(} S^{*} \gamma_{1}, \ldots, S^{*} \gamma_{r} . S K_{1}^{*}, \ldots, S K_{n}\right):=\sigma \cdot \Psi\left(\gamma_{1}, \ldots, \gamma_{r}, K_{1}, \ldots, K_{n}\right)$ for $\gamma_{i} \in \mathcal{L}^{*}$ and $K_{i} \in \mathcal{L}$.

For example, if a two-times contravaint $(i .2)$ means that where $<.>$ denote the respective $\gamma_{1}, \dot{\Theta} S^{*} \gamma_{2}>=\sigma\left(<\gamma_{1}, \Theta \gamma_{2}>\right)$ for all $\gamma_{1}, \gamma_{2} \in \mathcal{L}^{*}$ wenote the respective dualities in $\mathcal{L}$ and $\dot{\mathcal{L}}$. This yields

where $S^{T}: \hat{L}^{*}-L^{*}$ is the transpose of $S$ given by

$$
\check{\Theta}=S \Theta S^{T}
$$

$$
\sigma\left(<S^{*} \gamma, K>\right)=\left\langle\tilde{y}, S^{*} K>\right.\text {. }
$$
Observe that in this formula we used $S^{* T}=S^{-1}$. With the same ease other transfor
mation formulas may be explicitly detelmined (see [4)]. As a consequence of our invariant
definitions we find

Remark 7.1: The notions implectic, symplectic, closed and hereditary are invariant under
Lie-module isomorphisms. The most important Lie-module isomorphisms are given by variable transformations and these constitute an efficient tool for the construction of new compatible pairs of implectic
operators. Variable transformations: 
Let $M$ and $\hat{M}$ be $C^{\infty}$-manifolds and denote the respective manifold variables by $u$ and i. Assume that there is a function $u \rightarrow \bar{u}=T(u)$ such that $T$ and its inverse (denoted by $\tilde{T}$ ) are $C^{\infty}$. Let the meaning of $\mathcal{L}^{\prime}$ and $\mathcal{F}$ be as in the standard situation and consider the corresponding Lie module with respect to $M$. Then vector fields from $M$ to $\dot{M}$ are transformed by the variational derivative of $T$ :

$$
K(u) \rightarrow \tilde{K}(\tilde{u}):=T^{\prime}(\tilde{T}(\tilde{u}))[K(\tilde{T}(\tilde{u}))]
$$

and for scalars we define

$$
f(u) \rightarrow \tilde{f}(\tilde{u}):=f(\tilde{T}(\tilde{u})) .
$$

These transformations define a Lie-module isomorphism.

The most simple example for that isomorphism is when $M$ is a vector space and

$$
\vec{u}=\lambda u+a,
$$

where $\lambda$ is some scalar and $a$ some constant vector in $M$. Then we have that $S:=T^{\prime}=\lambda I d$ and we obtain

Remark 7.2: Under the substitution $u \rightarrow \lambda u+a$ (a a constant vector and $\lambda$ some scalar) it tach tensor the properties: implectic, symplectic, closed and hereditary are preserved.

\section{Example 7.3; Construction of compatible pairs}

For technical reasons, we now consider the space $\mathcal{S}_{-}$of $C^{\infty}$-functions $f$ in the real variable $\{\in \mathbb{R}$ having the property that $f$ and all its derivatives vanish at $-\infty$ faster than any ra:ional function and that at $+\infty$ all derivatives grow at most polynomially. As $S_{+}$we denote the corresponding space where the role of $-\infty$ and $+\infty$ has been interchanged. Between $S_{+}$and $S_{-}$we introduce an $L^{2}$ scalar product as in (3.9)

Sisefore, $D$ denotes differentiation with respect to $x$ and $D^{-1}$ denotes integration from

$$
\langle U, u\rangle=\int U(x) u(x) d x, \quad U \in \mathcal{S}_{+}, u \in \mathcal{S}_{-} .
$$

- $x$ to $x$. Let the manifold under consideration be $M=\mathcal{S}_{-}$. Observe that $D$ is implectic and invertible. We consider the variable transformation $\bar{u}=T(u):=u^{2}+u_{x}$ on $M$. Observe that, because of the bound the Implicit Finction Theorem. Tound $T^{\prime}(u)=2 u+D$ the inverse of $T^{\prime}(u)$ by solving the linear differential equation $T^{\prime}(u) z=g$ for given $g$ and unknown $z$. On $\mathcal{S}_{-}$this has a mique solution and the operator $T^{\prime}(u)^{-1}$ mapping $g$ into $z$ is

Starting with the implectic operator $D$ (with respect to the manifold variable $u$ ) we find by

$$
T^{\prime}(u)^{-1}=\exp \left(-2\left(D^{-1} u\right)\right) D^{-1} \exp \left(+2\left(D^{-1} u\right)\right) \text {. }
$$

ariable transformation (formula (7.4)) that

$$
\begin{aligned}
\dot{\Theta}(\bar{u}) & =T^{\prime}(u) D T^{\prime}(u)^{T} \\
& =(2 u+D) D(2 u-D) \\
& =D^{3}+2 D\left(u^{2}+u_{x}\right)+2\left(u^{2}+u_{x}\right) D
\end{aligned}
$$


Using the relation between $u$ and $\tilde{u}$ we see that

$$
\tilde{\Theta}(\bar{u})=D^{3}+2 D \bar{u}+2 \tilde{u} D
$$

is implectic as it was clajmed already in Example 3.5. Now performing the substitution for $a(x)=1$ ) as described in Remark $i .1$ we find that

$$
\left.\tilde{\Theta}(\tilde{u}+1)=D^{3}+2(D \tilde{u}+\tilde{u} D)+4 I\right)=\tilde{\Theta}(\tilde{u})+4 D
$$
is again implectic. Because $D$ is already known to be implectic we have that $D$ and $\dot{\Theta}$ are
compatible. Hence by Corollary 6.10

must be hereditary.

$$
\tilde{\Phi}=\tilde{\Theta} D^{-1}=D^{2}+2 D \bar{u} D^{-1}+2 \tilde{u}
$$

Using Theorem 6.11 we find that

$$
\bar{\Theta}_{2}(\tilde{u})=\tilde{\Phi}(\tilde{u}) \tilde{\Theta}(\tilde{u})
$$

again is implectic. We transform that back from the $\tilde{u}$-variable to the $u$-variable in order to obtain with (7.10) the following implectic operator:

Vow using $u-i$ we find with Remark 7.1 that

$$
\begin{aligned}
\Theta(u) & =T^{-1} \dot{\Theta}_{2}\left(T^{\prime-1}\right)^{T} \\
& =T^{\prime-1} \tilde{\Theta} D^{-1} \tilde{\Theta}\left(T^{\prime-1}\right)^{T} \\
& =T^{-1} T^{\prime} D\left(T^{\prime}\right)^{T} D^{-1} T^{\prime} D\left(T^{\prime}\right)^{T}\left(T^{\prime-1}\right)^{T} \\
& =D\left(T^{\prime}\right)^{T} D^{-1} T^{\prime} D \\
& =D(2 u-D) D^{-1}(2 u+D) D \\
& =-D^{3}+4 D u D^{-1} u D
\end{aligned}
$$

\[ (u)_{n i d v}=D^{3}+4 D u D^{-1} u D \]
is implectic. Using Remark $i: 2$ and $u \rightarrow(1 / 4) \sqrt{2}(2 u+1)$ we find that

$$
\begin{aligned}
& \Theta(u)_{n i k v}=D^{3}+4 D u D^{-1} u D \\
& 7.2 \text { and } u \rightarrow(1 / 4) \sqrt{2}(2 u+1) \text { we find } \\
& \text { Gardner }=\frac{1}{2}\left(\Theta(u)_{m k d V+\Theta(u)+D)}\right.
\end{aligned}
$$
is also implectic. here $\Theta(u)$ is the inplectir operator given in ( 7.11$)$ (only $\tilde{u}$ replaced by $u$.
Since $\Theta(u)$ and $D$ are compatible $\Theta(u)+D$ is again implectic and $\Theta$ mKav and $\Theta(u)+D$
mist be compatible. Taking now servation 6.5) and substituting $u \rightarrow(2 u d V+3 / 4(\Theta(u)+D)$ (which is implectic by $0 b$. Herce $\Theta_{m h d l}$ and $D$ are comg $u \rightarrow(2 u-3)$ we find that $\Theta_{m k d}+(3 / 4) D$ is implectic

Observation 7.4: If the duality between function spaces is taken to be (3.9) then thi
differential operators

$$
\begin{aligned}
\Theta_{0} & =D \\
\Theta_{K d V} & =D^{3}+2 D u+2 u D \\
\Theta_{m K d V} & =D^{3}+4 D u D^{-1} u D
\end{aligned}
$$


are implectic. Every tuo of these are compatible.

\section{Example 7.5: Conserved quantities for the $\mathrm{KdV}$}

Consider the situation of Example 3.5. where the operator

$$
\Theta_{K d V}=D^{3}+2 D u+2 u D
$$

was introduced. Taking into acconnt the compatibility between the implectic operators $D$ and $\Theta_{K d V}$ (as just proved) we get from Corollary 6.10 that

$$
\Phi=\Theta D^{-1}=D^{2}+2 D u D^{-1}+2 u,
$$

(bihamKdV)

is hereditary. As a consequence (Theorem 6.11) we have that the

$$
\gamma_{n+1}=D^{-1} K_{n+1}=D^{-1} \Phi(u)^{n} K(u) \text {, where } K(u)=6 u u_{x}+u_{x x x}
$$

are closed co-vector fields. These fields are invariant for any of the flows $u_{t}=K_{n}(u)$. Hence (Lemma 5.5) all

$$
I_{n}(u)=\int_{0}^{1}<\gamma_{n}(\lambda u), u>d \lambda=\int_{0}^{1} \int_{\mathbb{R}} \gamma_{n}(\lambda u(x)) u(x) d \lambda d x
$$

are conserved quantities for every one of these flows, especially for the KdV. In addition, one easily sees that all these quantities commute with respect to the Poisson brackets defined by either $\Theta_{K d V}$ or $D$

\section{Example 7.6: Further systems}

Using the compatible pairs we have up to now, and more which we generate easily by 作 example

$$
\begin{aligned}
\Phi_{m K d V} & =\Theta_{m K d V} D^{-1} \\
\Phi_{\text {Gardner }} & =\Theta_{\text {Gardner }} D^{-1} \\
\Phi_{\text {sineGordon }} & =2 \Phi_{m K d V}^{-1} \\
\Phi_{B B M-\{i k e}(v) & =\left(I-D^{2}\right)^{-1} \Phi_{K d V}\left(v-v_{x x}\right) \\
\Phi_{s G-K d V} & =\Phi_{h d V}^{-1} \Phi_{\text {sineGordon }}
\end{aligned}
$$

The hereditary nature of these operators is easily seen from compatibility of known pairs. For example, take $\Phi_{s G-K d V}$ then this can be written as $\Theta_{K d V} \Theta_{m K d V}^{-1}$, and must be hered itary since these two are compatible. For $\Phi_{B B M-l i k e}(v)$ we use the compatibility of $\Theta_{K d v}$ and of $D-D^{3}$, and then we performed a variable transformation $u=v-v_{x x}$.

Since none of these operators depend explicitly on $x$ we find that they are all invariant with respect to the special vector field $u_{x}$. So for the equations

$$
u_{t}=\Phi(u) u_{x}
$$


we find (by application of Theoren 6.1) infinitely many symmetry group generators

$$
K_{n}(u)=\Phi^{n}(u) u_{x}
$$

Let us list thesc equations (following the above order)

$$
\begin{gathered}
u_{t}=u_{x x x}+6 u^{2} u_{x} \\
u_{t}=u_{x x x}+6 u^{2} u_{x}+6 u u_{x}+u_{x} \\
u_{t}=\sin \left(2 \int_{-\infty}^{x} u(\xi) d \xi\right) \\
v_{t}-v_{x x t}=v_{x x x}-2 v v_{x x x}-4 v_{x} v_{x x}+6 v v_{x} \\
v_{x t}=2 v_{x x} \cos (2 v)+4\left(v_{x}-v_{x}^{2}\right) \sin (2 v)+2 v_{x x} \int_{-\infty}^{x} \sin (2 v(\xi)) d \xi
\end{gathered}
$$

(modified $K d V$ )

(Gardner eq.)

(potential_sG eq.)

(BBM like eq.)

$($ ( $) d V . s G \in q)$

In the case of the last equation ( $K d V \_s G$ ), we have performed an additional variable transformation $u=v_{x}$. The equation (potential_sG) is connected to so called sine_Gordon space of independention first $u=v_{x}$ and performing then a 45-degree rotation in the space of independent variables yields

$$
v_{\xi \xi}-n_{\eta \eta}=\sin (2 v) \text {. }
$$

(sine_Gordon equation)

Most of these equations are well known from the literature. They all have a bi-hamiltonian formulation if the solution manifold is suitably chosen such that these operators are well because they are then generated by a hererated by this recursion mechanism are all closed pair of implectic operators. For example hereditary operator, which stems from a compatible (modifiedKdV) the compatible example, in case of the modified Korteweg-de Vries equation itself has the form

Furthermore

$$
u_{t}=u_{x r x}+6 u^{2} u_{x}=\Theta_{m K d V}(u) \nabla \frac{1}{2} \int_{\mathbb{R}} u^{2}(\xi) d \xi .
$$

Application of Theorem 6.11 then shows $D^{-1}\left(u_{x x s}+6 u^{2} u_{x}\right)=\nabla \int_{\mathbb{R}}\left(-u_{x}^{2}(\xi)+\frac{1}{2} u^{4}(\xi)\right) d \xi$.

$$
I_{n}(u)=\int_{0}^{1} \int_{\mathbb{R}} \gamma_{n}(\lambda u(x)) u(x) d x d \lambda=\int_{0}^{1} \int_{\mathbb{R}}\left(D^{-1} \Theta_{m \kappa d V}(\lambda u(x))\right)^{n} u(x) d \lambda d x
$$

are also conserved quantities for modified Korteweg-de Vries equation. Observe that these since that equation is generated by the potential sine Gordon equation (potential_s eq.) go through for the other equations the inverse of the operator $\Phi_{m k d V}$. Similar arguments

tions given above.

way exhausted by these that the applications of the theory presented in this paper are in no equation, two-component by Fokas and Santini $([2],[24],[23][22)$ chains, and the bi-hamiltonian formulations given last examples are interesting in $[23],[22)]$ for equations in two independent variables. These methods as introduced in Sections 4,5 and they require the full generality of notions and 


\section{Integrability and Solitons}

Let us briefly review:

Complete integrability in the finite dimensional case:

The hamiltonian flow

$$
u_{t}=\Theta(u) \nabla H(u) .
$$

on a $2 N$-dimensional manifold $M$ with invertible implectic operator $\Theta$ is called completely integrable if it admits $N$ conserved quantities $I_{1}:=H, I_{2}, \ldots, I_{N}$ such that the corresponding symmetry group generators $\Theta \nabla I_{1}, \Theta \nabla I_{2}, \ldots, \Theta \nabla I_{N}$ commute. Furthermore these fields are required to be linearily independent at each manifold point. These $I_{1}, I_{2}, \ldots, I_{N}$ are called action variables.

Observation 8.1:

In this case one can find $N$ closed and pairwise commuting vector fields $A_{1}, \ldots, A_{N}$ such that

$$
L_{A_{1}} I_{j}=\delta_{i j} I_{i}
$$

or. by use of (4.16) and Theorem 4.5

$$
\left[A_{i}, \Theta \nabla I_{j}\right]=\delta_{i j} \Theta \nabla I_{i}
$$

The $\Theta \nabla I_{i}$ are called action fields and the $A_{i}$ are called the conjugate angle fields).

The proof of this statement is technically involved, so we will only give a brief sketch. The arguments are an adaption of [16, page 28].

Proof :

STEP 1:

First one shows that around each manifold point $u_{0}$ coordinates $\left\{I_{1}, \ldots, I_{N}, Q_{1}, \ldots, Q_{N}\right\}$ can First one shows that around each manifold point $u_{0}$ coordinates $\left\{I_{1}, \ldots, I_{N}, Q 1, \ldots, Q\right.$ in the following
be chosen such that $J(u):=\Theta(u)^{-1}$ is constant in that chart. This is done in the
way:

Represent the manifold around $u_{0}$ by some open ball in a vector space $E$ with coordinates $\left\{I_{1} \ldots, I_{N}, \tilde{Q}_{1}, \ldots \tilde{Q}_{N}\right\}$. Then consider the operators $J(u)$ and $J_{0}(u):=J\left(u_{0}\right)$ and take a feformation $J_{t}(u):=J(u)+t\left(J_{0}(u)-J(u)\right), 1 \geq t \geq 0$ from $J$ to $J_{0}$. Observe that $J_{\varepsilon}\left(u_{0}\right)=J_{0}\left(u_{0}\right)$ is invertible for all $t$ with $1 \geq t \geq 0$. The openness of the set of isomorphisms shows that there is a ball $B$ around $u_{0}$ such that $J_{t}(u)$ is invertible for all $u \in B$ and $l \geq t \geq 0$. The inverse of $J_{t}(u)$ we denote by $\Theta_{t}(u)$. Since $\left(J_{0}(u)-J(u)\right)$ is closed we find by the Poincaré lemma a 1-form $\gamma$ in $B$ such that $\left(J_{0}(u)-J(u)\right)=d \gamma$ and $\gamma\left(u_{0}\right)=0$. Now take the $t$-dependent vector field $h(t, u)=\Theta_{t}(u) \gamma$ and consider the equation

$$
v_{t}=K(t, v)
$$

in $B$. Since $K(t=0, u)=0$ we can assume (by eventually restricting $B$ again) that (8.3) has a unique solution for all $1 \geq t \geq 0$ and all $u \in B$. Define $\varphi(u)$ to be the solution of (8.3) for $t=1$ and initial condition $v(t=0):=u$ and let $\tilde{I}_{i}(u)=I_{i}(\varphi(u)), \bar{Q}_{i}(u)=Q_{i}(\varphi(u)), i=$ 
$1, \ldots, N$. Observe that $\tilde{I}=I$ and that $J(u)$ now is constant in the chart given by these new coordinates.

STEP 2:

Consider the constant $J(u)$ as constructed above in the vector space given by the coordinates $\left\{I_{1}, \ldots, I_{N}, Q_{1}, \ldots, Q_{N}\right\}$ and endow that space with the usual Euclidean metric of $\mathbb{R}^{2 N}$. Since $J(u)$ is invertible and antisymmetric its matrix representation must be of the form

$$
\left(\begin{array}{cc}
0 & S \\
-S & 0
\end{array}\right)
$$

where $S$ is invertible and symmetric. Hence by a change of basis among the $Q$ 's we can assume that $S$ is the $N \times N$ identity matrix. Finally, taking $A_{i}=-\Theta \nabla\left(I_{i} Q_{i}\right)$ we locally find the desired vector fields.

STEP 3:

We observe that different local realizations of (8.1) differ on the overlap of their domain only by a suitable combination of the $\Theta \nabla I_{i}$, hence by globally defined vector fields. This property allows us to patch the $A_{i}$ from one chart to the next so that they coincide on the overlap. Hence we can define them globally.

Observe that locally potentials for the $A_{i}$ exist, let us call them $Q_{i}$. Then (8.1) inlplies that for every of the flows $u_{t}=\Theta(u) \nabla I_{n}$ the $Q_{m}, n \neq m$ are conscrved quantities. whereas $Q_{n}$ changes with $t$ but is the absolute part of the time-dependent conserved quall tity $Q_{n}-t I_{n}$. So taking these coordinates we arive at

Observation 8.2: On some $2 N$-dimensional manifold, endowed with the invertible implec. tic operator $\Theta$, let there be given $N$ pairwise commuting scalar fields $I_{1}, \ldots, I_{N}$ (commuting with respect to the corresponding Poisson brackets). Then around each point there are locit coordinates $\left\{I_{1}, \ldots, I_{N}, Q_{1}, \ldots, Q_{N}\right\}$ such that the hamillonian flows $u_{t}=\Theta(u) \nabla I_{n}$ are liner in these coordinates so that all but $Q_{n}$ are invariant and that the action on the $Q_{n}$ is such that this grows linear with $t$. $J_{2} \mathcal{L}-\mathcal{L}^{-}$

Now we use the vector fields from Observation 8.1 to define the following operator
C $^{-}$

$$
J_{2}=\sum_{i=1}^{N} \Theta^{-1} A_{i} \circlearrowleft d I_{i}-d I_{i} \otimes \Theta^{-1} A_{i} .
$$

Obviously this is an antisymmetric tensor and it is closed because both $d I_{i}$ and $\Theta^{-1} A_{i}$ art closed. So it may serve as a symplectic operator and one easily finds that

is hereditary. lising the relations (8.1) we see right away that

$$
\Phi:=\Theta J_{2}
$$

$$
\begin{aligned}
J_{2} \Theta \nabla I_{i} & =I_{i} \nabla I_{i} \\
J_{2} A_{i} & =-I_{i} \Theta^{-1} A_{i}
\end{aligned}
$$

and that therefore the eigenvalues of $\Phi$ are given by the action variables. So we have 
Observation 8.3: For a finite dimensional completely integrable system in $2 \mathrm{~N}$-dimensional space, with action varaibles $\left\{I_{1}, \ldots . I_{N}\right\}$ there aluays erists a hereditary operator $\Phi$ such that its spectrum is doubly degenerate. The eigenvalues are given by the action variables and the corresponding eigenvectors are the action fields and their conjugate angle fields.

Of course, the converse is also true: Whenever on a $2 N$-dimensional manifold we have a hereditary opcrator $\Phi$ with doubly degenerate spectrum and some implectic openator $\theta$ such that $\Theta \Phi$ is closcd, then when the the gradients of the eigenvalues are mapped with $\theta$ onto vector fields they form a commuting algebra of vector fields (consequence of Remark 6.3). Hence any dynamic of system given by any linear combination of these vector fields thust be complctely integrable.

Now let us return to the general situatjon of infinitely dimensional manifolds. Here the notion of complete integrability has not yet been definitely defined in the literature. Often completely integrable if it admits an infinite dimensional abelian of hamiltonian fields. This of course, is a somehow loose definition since it is easy to construct situations where such a symmetry group does not suffice to guarantee a parametrization by action and angle variables. Instead of attempting here a general defintion we shall show that, under reasonable conditions, the existence of a compatible bi-hamiltonian pair leads to complete integrability on finite dimensional reductions given by the corresponding symmetry group generators. We will give a survey on the results which can be obtained in that direction, for details the reader is referred to [8]

Let us recall the situation we considered before. On a suitable manifold $M$ the evoution equation $u_{t}=K_{1}(u)$ where $u=u(x, t) \in M$ was considered. We assume that there a hereditary recursion operator $\boldsymbol{\Phi}(u)$ generated out of a compatible hamiltonian pair

$$
\Phi(u)=\Theta_{2}(u) \Theta_{1}^{-1}(u)=: \Theta_{2}(u) J(u) .
$$

As shown the operator $\Phi$ then generates a hierarchy of pairwise commuting infinitesimal simmetry group generators

$$
K_{n+1}(u):=\Phi^{n}(u) K_{1}(u)
$$

for the evolution equation under consideration.

In addition to what we assumed until now we require furthermore the existence of a scaling symmetry $\tau_{0}(u)$. By that we mean:

$$
\left[\tau_{0}, K_{1}\right]=(\varrho+1) K_{1}
$$

and

$$
L_{T_{0}} \Phi=\Phi
$$

As a consequence of the scaling property the recursive application of $\Phi$ on $\tau_{0}$ produces a second hierarchy of vector fields, the so-called mastersymmetries $[6] \tau_{n}=\Phi^{n} \tau_{0}$ such that the ond hierarchy of vector fields, the so-called mastersymmetries $[0] \tau_{n}=\Phi^{n} \tau_{0}$ sthes the mastersymmetries
following commutator relations hold between the symmetries $K_{n}$ and the mas
$\tau_{r}$

$$
\left[K_{n}, K_{m}\right]=0,\left[\tau_{n}, K_{m}\right]=(m+\varrho) K_{n+m}, \quad\left[\tau_{n}, \tau_{m}\right]=(m-n) \tau_{n+m} .
$$


Indeed, these commutator relations are a simple consequence of the hereditary property of $\Phi$. One should observe that the relation $\left[\tau_{n}, K_{m}\right]=(m+\varrho) K_{n+m}$ is equivalent to the fact that $\tau_{n}+(m+\varrho) K_{n+m}$ is a time-dependent symmetry group generator of $u_{t}=K_{m}(u)$. A Lie algebra consisting of $\tau$ 's and $K$ 's fulfilling $(8.9)$ is called a hereditary algebra. These scaling symmetries exist for almost all popular soliton equations, and even in those cases where a scaling symmetry or a hereditary cannot be found one can nevertheless construct a suitable hereditary algebra. For example, in the $\mathrm{KdV}$ case $\tau_{0}(u)=\frac{1}{2} x u_{x}+u$ is the scaling symmetry, and for the $m \mathrm{KdV}$ and the potential sine-Gordon one finds $\tau_{0}(u)=x u_{x}+u$.

From the invariance of the symmetry group generators one finds that the submanifold (see for example [19])

$$
M_{N}=\left\{u \mid \text { there exists } \alpha_{n} \text { such that } \sum_{n=0}^{N} \alpha_{n} K_{n}=0\right\}
$$

is invariant under any of the flows $u_{t}=K_{n}(u)$, in particular under $u_{t}=K_{\mathrm{I}}(u)$. This manifold is called the manifold of $N$-soliton solutions. For the $\mathrm{KdV}$ typical two-and three-solitons are given in figures 3 and $4^{9}$.

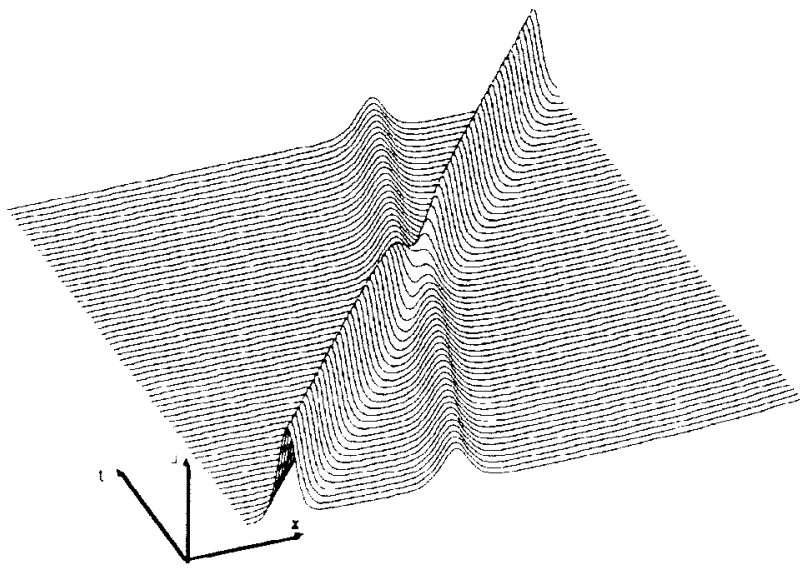

Fig. 3: T'wo-soliton of the KdV

\footnotetext{
${ }^{9}$ I am indebted to Thorsten Schulze for plot ting these figures.
} 


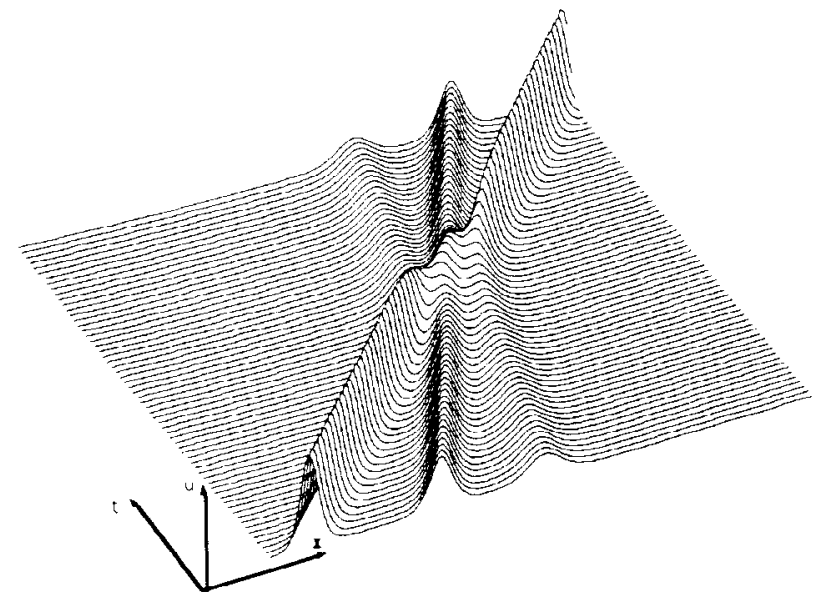

Fig. 4: Two-soliton of the $\mathrm{KdV}$

In case when the boundary conditions at infinity for possible solutions $u$ are chosen in such a way that the resulting manifold $M_{N}$ has dimension $2 N$ then by a lengthy but simple analysis [8] (mainly of the hereditary structure of $\Phi$ ) one obtains from this structure the following result

\section{Theorem 8.4:}

(1) For all $r, p \in N_{0}$ we have the following representation of the tangent space $T_{1} M_{N}$ of $M_{N}$ at the point $u$

$$
T_{u} M_{N}=\operatorname{span}\left\{K_{T}, h_{r+1}, \ldots, K_{r+N-1}, \tau_{p}, \tau_{p+1}, \ldots, \tau_{p+N-1}\right\} .
$$

12) Whenever the $\alpha_{n}$ are the coefficients given by (8.10) (to define the manifold point $u$ ) then the following hold

(i) For all $r \in N_{0}$ we have the following identities on $M_{N}$ :

$$
\sum_{n=0}^{N} \alpha_{n} K_{n+r}=0 \text { and } \sum_{n=0}^{N} \alpha_{n} \tau_{n+r}=0 .
$$

(ii) The discrete eugenvalues $c_{1}, \ldots, c_{N}$ of $\Phi$ are given as the zeros of the characteristic polynominal $P(\xi)=\sum_{n=0}^{N} \alpha_{n} \xi^{n}$.

(iii) The corresponding eigenstates are $\bar{V}_{i}=\Pi_{i}(\Phi) K_{0}$ and $\bar{W}_{i}=\Pi_{i}(\Phi) \tau_{0}$, where $\Pi_{i}(\xi)=P(\xi) /\left(\xi-c_{i}\right)$. 
As a direct consequence of Theorem 8.4 we obtain that the recursion operator $\Phi$ leaves the tangent space $T_{u} M_{N}$ of the reduced manifold invariant. Hence, the restriction $\Phi:=\Phi_{\text {jred }}$ of $\Phi$ to $M_{N}$ is a linear operator on a finite dimensional space. This operator $\bar{\Phi}$ has the properties listed below in:

\section{Observation 8.5:}

(1) $\bar{\Phi}$ is invertible and can be written as $\bar{\Phi}=\ddot{\Theta}_{2} \bar{\Theta}_{1}^{-1}$ where $\bar{\Theta}_{1}, \bar{\Theta}_{2}$ are a compatible pair of implectic opcrators.

(2) The eigenvalues $c_{1}, \ldots, c_{N}$ of $\bar{\Phi}$ are doubly degeneratcd.

(3) Renorming the eigenstates $\tilde{V}_{i}, \tilde{W}_{i}$ leads to eigenstates $V_{i}$ and $W_{i}$ which are hamiltonian vector fields w.r.t. $\bar{\Theta}_{1}$ and $\bar{\Theta}_{2}$.

(1) The eigenstates $V_{i}$ and $W_{i}$ fulfil the commutator relations

$$
\left[V_{i}, V_{j}\right]=0=\left[W_{i}, W_{j}\right] \quad, \quad\left[V_{i}, W_{j}\right]=\delta_{i j} V_{j} .
$$

These last two results show that the finite dimensional reductions, given by those nembers of the abelian symmetry group which is generated by the bi-hamiltonian formulation is, under suitable boundary conditions al infinity, the same situation as we found in the completely integrable finite dimensional case.

Since the eigenstates $V_{i}, W_{i}$ are hamiltonian vector fields and since they fulfill the canonical commutator relations (8.9), their potentials can be interpreted as action/angle variables for the flow induced by (1.1) on $M_{N}$.

Although all our considerations were of a purely algebrajc nature we should remark that in most cases which are relevant from the physical viewpoint the $N$-soliton solutions (with vanishing boundary conditions at infinity) are those solutions which decompose into
$V$ single waves for $t \rightarrow \pm \infty$

$$
u_{N} \cong \sum_{i=1}^{N} s_{i}\left(x+c_{i} t+q_{i}\right) .
$$

This can be seen for the KdV from Figures 4 and 5. By comparison with the asymptotic operator.

Observation 8.6: Taking the partial derivatives of $u_{N}$ w.r.t. the asymptotic data

$$
\frac{\partial u_{N}}{\partial q_{i}} \text { and } \frac{\partial u_{N}}{\partial c_{i}}
$$

one obtains eigenstates of the recursion operator $\bar{\Phi}$ for the eigenvalue $c_{i}$. The function $\partial u_{N} / \partial q_{i}$ then is the vector field corresponding to the action variable and this is called the 


\section{Hamiltonian Structure and Integrability}

interacting soliton $[y]$.

In case of the kdV equation a plot of such quantities is easily obtained (see Figure 5)

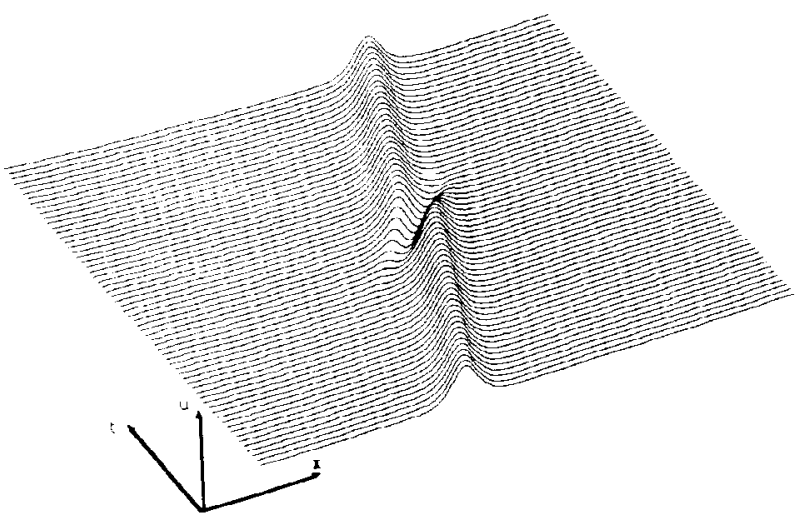

Fig. 5: Interacting soliton of the $\mathrm{KdV}$

A corresponding conjugate eigenstate for the $\mathrm{KdV}$ is obtained by taking the derivative of the field function $u_{N}$ with respect to the parameters given by an eigenvalue of the recursion operator. 


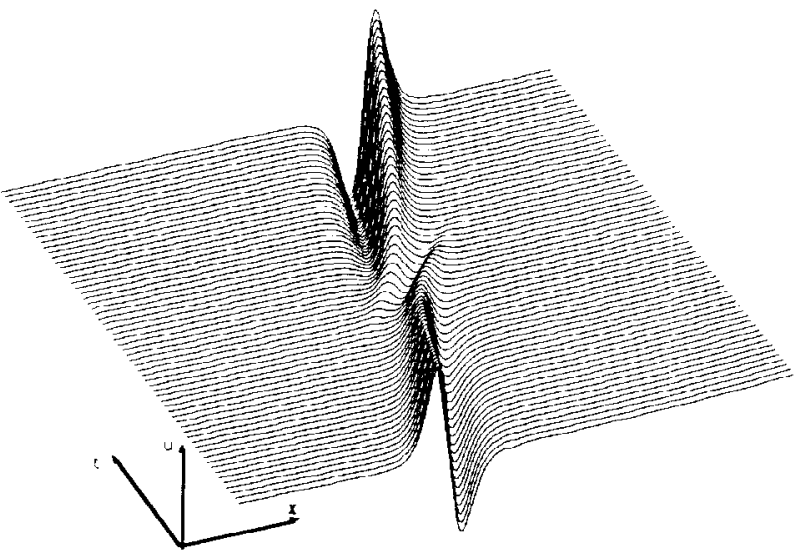

Fig. 6: Derivative of Angle-variable density of the $\mathrm{KdV}$

It should be remarked that the field functions given by these plots themselves satisfy nonlinear equations which have a compatible bi-hamiltonian formulation $[7]$

\section{References}

[1] A. Dick: Emmmy Noether, Birkhäuser-Verlag, Basel, 1970

[2] A.S. Fokas and P.M. Santini: The Recursion Operator of the Kadomtsev-Petviashribl equation and the squared eigenfunctions of the Schrodinger Operator, Studies in Appl
Matl.. 75. p.179-186. 1986

[3] B. Fuchssteiner: Application of Hereditary Symmetries to Nonlinear Evolution equations. Nonlinear Analysis TMA, 3, p.849-862, 1979

[4] A. S. Fokas and B. Fuchssteiner: Bäcklund Transformations for Hereditary symmetries Nonlinear Analysis TMA, 5, p.423-432, 1981

[5] B. Fuchssteiner and A. S. Fokas: Symplectic Structures, Their Bäcklund Transformations and Hereditary Symmetries, Physica, 4 D, p.47-66, 1981

[6] B. Fuchssteiner: Mastersymmetries, Higher-order Time-dependent symmetries and conserved Densities of Nonlinear Evolution Equations, Progr. Theor.Phys., 70, p.1508-
1522,1983 
[i] B. Fuchssteiner: Solitons in Interaction, Progress of Theoretical Physics, 78, p.10221050, 1987

[8] B. Fuchssteiner and G. Oevel: Geometry and action-angle variables of multisoliton systems, Reviews in Mathematical Physics, 1, p.415-479, 1990

[9] B. Furhssteiner: Compatibility in abstract algebraic Structures: Application to nonlinear systems, Paderborn, preprint, 1990

10] L.M.Gelfand and 1.Y. Dorfman: Funktsional'nyi Analiz i Ego Prilozheniya, Hamiltonian Operators and Algcbraic Structures related to them, 13, p.13-30, 1974

11] I.M.Gelfand and I.Y. Dorfman: Funktsional'nyi Analiz i Ego Prilozheniya, The Schouten bracket and Hamiltonian operators, 14, p.71-74, 1980

12] I.M.Gelfand and I.Y. Dorfman: Funktsional'nyi Analiz i Ego Prilozheniya, Hamiltonian Operators and Infinite-Dimensional Lie-Algebras, 15, p.23-40, 1981

[3] D. J. Korteweg and G. De Vries: On the change of form of long waves advancing in a rectangular canal, and a new type of long stationary waves, Philos.Mag.Ser. 5, 39, p.422-443, 1895

14] F. Magri: A simple model of the integrable Hamiltonian equation, J.Math.Phys., 19 , p.1156-1162, 1978

1.5] F. Magri: A Geometrical Approach to the Nonlinear Solvable equations, Nonlinear Evolution equations and Dynamical Systems (M. Boiti, F. Pempinelli, G. Soliani eds.) Springer Verlag, p.233-263, 1980

(6) J. Marsden: Applications of Global Analysis in Mathematical Physics, Publish or Perish, Inc., 2, Boston, 1970

17] E. Nelson: Tensor Analysis, Princeton University Press, Princeton N.J, 1967

18. E. Noether: Invariante Variationsprobleme, Nachr.v.d. Gesellschaft d.Wiss. zu Göttingen, p.235-257, 1918

19] S.P. Novikov, S. V. Manakov, L. P. Pitaevskii and V. E. Zakharov: Theory of Solitons The Inverse Scattering Method. Consultants Bureau, New York-London, 1984

20. P.J. Olver: Evolution Equations possessing infinitely many symmetries, J.Math.Phys.. 18,1977 p.1212-1215,

$21 \mathrm{C}$ C. Rebbi and G. Soliani: Solitons and Particles, World Scientific, Singapore, 1984

22] P.M. Santini and A.S. Fokas: Recursion Operators and Bi-Hamiltonian Structures of $2+1$ dimensional systems, in: Topics in Soliton Theory and Exactly solvable Nonlinear equations (eds: M. Ablowitz, B. Fuchssteiner, M. Kruskal) World Scientific Publ. Singapore, 1987 p.1-19, 
[23] P.M. Santini and A.S. Fokas: Recursion Operators and Bi-Mamiltonian structures in Multidimensions. I, Commun. Math. Phys., 115, p.375-419, 1988

[24] P.M. Santini, A.S. Fokas: Recursion Operators and Bi-Hamiltonian structures in Mutidimensions. II, Comm. Math. Phys., 116, p.449-474, 1988

[25] B. Schutz: Geometrical Methods of Mathematical Physics, Cambridge University Press. Cambridge-London-La. Rochelle-New York-Melbourne-Sidney, 1980

[26] S. Yamamuro: Differential Calculus in Topological Linear Spaces, 374, Springer Verlag. Berlin-Heidelberg-New York, 1974

[27] S. Yamamuro: A theory of differentiation in locally convex spaces, AMS, Providence. Rhode Island, 1979 